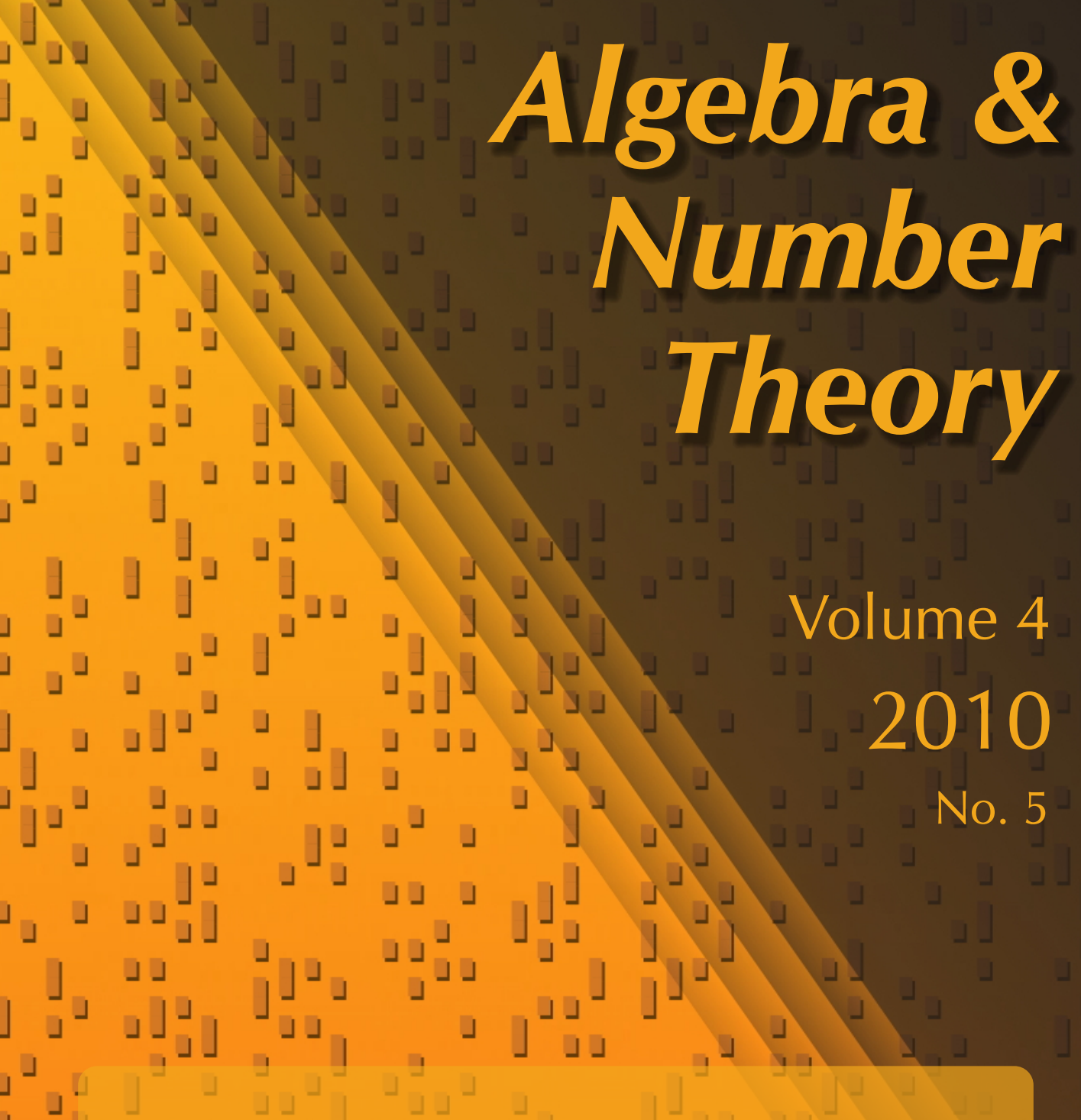

The Manin constant of elliptic curves over function fields Ambrus Pál

\lrcorner

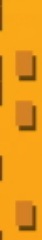

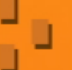
\lrcorner$\lrcorner$ 


\title{
The Manin constant of elliptic curves over function fields
}

\begin{abstract}
Ambrus Pál
We study the $p$-adic valuation of the values of normalised Hecke eigenforms attached to nonisotrivial elliptic curves defined over function fields of transcendence degree one over finite fields of characteristic $p$. We derive upper bounds on the smallest attained valuation in terms of the minimal discriminant under a certain assumption on the function field, and provide examples to show that our estimates are optimal. As an application of our results, we prove the analogue of the degree conjecture unconditionally for strong Weil curves with squarefree conductor defined over function fields satisfying the assumption mentioned above.
\end{abstract}

\section{Introduction}

Notation 1.1. Let $F$ denote the function field of $\mathscr{C}$, where the latter is a geometrically connected smooth projective curve defined over the finite field $\mathbb{F}_{q}$ of characteristic $p$. Let $\mathbb{A}$ denote the ring of adèles of $F$, and let $\mathrm{GL}_{2}$ denote the group scheme of invertible two-by-two matrices. Let $E$ be a nonisotrivial elliptic curve defined over $F$. Then we may associate a cuspidal automorphic representation of $\mathrm{GL}_{2}(\mathbb{A})$ to $E$ as follows. Let $E_{\bar{F}}$ denote the base change of $E$ to the separable closure $\bar{F}$ of $F$. For every prime $l$ different from $p$, one may attach to the étale cohomology group $H^{1}\left(E_{\bar{F}}, \mathbb{Q}_{l}(1)\right)$, considered as a representation of the absolute Galois group of $F$, an irreducible cuspidal automorphic representation $\rho_{E}$ with trivial central character via the Langlands correspondence. As the notation indicates, this representation is independent of the choice of $l$.

Let $V_{E}$ denote the irreducible constituent of the space of cuspidal automorphic forms on $\mathrm{GL}_{2}(\mathbb{A})$ that realises the representation $\rho_{E}$. Then there is a distinguished element $\psi_{E}$ of $V_{E}$ that we will call the normalised Hecke eigenform attached to $E$. It is characterised by the fact that it is invariant under the action of the Hecke congruence group of level $\mathfrak{n}$, where $\mathfrak{n}$ denotes the conductor of the elliptic curve $E$,

MSC2000: primary 11G05; secondary $11 \mathrm{G} 40,14 \mathrm{~F} 30$.

Keywords: elliptic curves, Hecke eigenforms, degree conjecture.

The author was partially supported by the EPSRC grant P19164. 
and its leading Fourier coefficient is 1. (For an explanation of these concepts as well as an explicit description of the Hecke eigenform, see Section 2.) By a classical theorem of Harder, the automorphic form $\psi_{E}$ takes only finitely many values. On the other hand, it is easy to see that it takes only rational values. Hence there is a unique positive rational number $c(E)$ such that the subgroup of $\mathbb{Q}$ generated by the values of $\psi_{E}$ is equal to $c(E) \mathbb{Z}$.

Proposition 1.2. There is a natural number $m(E)$ such that $c(E)=p^{-m(E)}$.

It is natural to guess that $m(E)$, which we will call the Manin constant of $E$, is always zero. Although this hypothesis is frequently made (sometimes implicitly) in the literature (see for example [Papikian 2005; 2007; Rück and Tipp 2000]), it is actually false. One of the aims of this paper is to exhibit many cases when $m(E)$ is not zero. Because the Manin constant could be nonzero, many formulas in the literature have to be corrected to include this nontrivial factor. Hence the latter is a very interesting isogeny invariant of the elliptic curve, and therefore it is desirable to compute it, or at least to give upper bounds, in terms of more well-known invariants. This is the other major aim of this paper. (This problem has been already studied in [Tan 1993]; see Remark 7.9). We will also discuss the implication of our results in connection with one of the formulas mentioned above.

We now formulate the main results of this paper. For every $E$ as above, let $\Delta_{E}$ denote the discriminant of a relatively minimal elliptic surface $\mathscr{E} \rightarrow \mathscr{C}$ whose generic fibre is $E$. Then $\Delta_{E}$ is an effective divisor on the curve $\mathscr{C}$. Moreover, let $g$ denote the genus of $\mathscr{C}$, and let $d$ be the positive integer such that $q=p^{d}$. We will show:

Theorem 1.3. Assume that $p$ does not divide the order of $\operatorname{Pic}_{0}(\mathscr{C})\left(\mathbb{F}_{q}\right)$. Then

$$
m(E) \leq d\left(\frac{1}{12} \operatorname{deg}\left(\Delta_{E}\right)+g-1\right),
$$

and the two sides of the inequality above are equal when the elliptic surface $\mathscr{E}$ is ordinary in dimension 2.

The condition on $\operatorname{Pic}_{0}(\mathscr{C})\left(\mathbb{F}_{q}\right)$ in the theorem above is satisfied, for example, when $\mathscr{C}$ is a rational curve or a supersingular elliptic curve. Moreover, the moduli space of smooth projective connected curves of genus $g$ with $p$-rank zero is a variety of dimension $2 g-3$ over $\overline{\mathbb{F}}_{p}$ when $g \geq 2$ and $p$ is odd [Faber and van der Geer 2004, Theorem 2.3 and Proposition 2.7]. Hence there are plenty of curves satisfying this condition. It is natural to expect that most elliptic surfaces are ordinary in dimension 2 (for a precise formulation of this conjecture, see Remark 6.12). In particular, our estimate in Theorem 1.3 should be the best possible (at least if we want to make one in terms of the discriminant). We can verify the ordinariness condition in many cases. The following result is just a sample of what can be proven with our methods. 
Theorem 1.4. Let $p$ be a prime number and let $n$ be a positive integer such that $n \mid p-1$ and $6 \mid n$. Let $E$ be the elliptic curve defined over the rational function field $F=\mathbb{F}_{p}(T)$ by the Weierstrass equation

$$
y^{2}+x y=x^{3}-T^{n} .
$$

Then $E$ is not isotrivial and

$$
m(E)=\frac{1}{6} n-1=\frac{1}{12} \operatorname{deg}\left(\Delta_{E}\right)-1 .
$$

The basic strategy of the proof of Theorem 1.3 is to relate the Manin constant to the $p$-adic valuation of coefficients of $L$-functions of $E$. The key tools in estimating the latter are a mild equivariant extension of Katz's conjecture relating the Newton and Hodge polygons and a theorem of Chinburg computing the refined equivariant Euler characteristic of the de Rham complex of varieties equipped with tame group actions in terms of $\epsilon$-constants. The proof of Theorem 1.4 is closely related. In fact, the reason why it is particularly convenient to work with those elliptic curves that appear in the theorem is that Ulmer [2002] computed their Hasse-Weil $L$-functions rather explicitly.

In the rest of the introduction we describe the application of Theorem 1.3 in this paper, which was the main motivation for our investigations. Fix a closed point $\infty$ of $\mathscr{b}$ and assume that $E$ has split multiplicative reduction at $\infty$. Then $\mathfrak{n}=\mathfrak{m} \infty$ for an effective divisor $\mathfrak{m}$ on $\mathscr{b}$; here and throughout we write the addition of divisors multiplicatively. Let $A$ denote the ring of rational functions on $\mathscr{C}$ regular away from $\infty$, and let $X_{0}(\mathfrak{m})$ denote the unique smooth projective curve over $F$ that contains the affine Drinfeld modular curve $Y_{0}(\mathfrak{m})$ parametrising Drinfeld $A$ modules of rank two of generic characteristic with Hecke level $\mathfrak{m}$-structure as a dense open subscheme. Then there is a nontrivial map $\pi: X_{0}(\mathfrak{m}) \rightarrow E$ of curves defined over $F$. We say that $E$ is a strong Weil curve if the modular parametrisation $\pi$ above can be chosen so that the kernel of the map induced by $\pi$ via Albanese functoriality is smooth and connected in the Jacobian of $X_{0}(\mathfrak{m})$. In this case we say that $\pi$ is optimal. Up to isomorphism, there is exactly one strong Weil curve in the isogeny class of $E$. With the help of Theorem 1.3 and the Pesenti-Szpiro inequality, we will show:

Theorem 1.5. Assume that $p$ does not divide the order of $\operatorname{Pic}_{0}(\mathscr{C})\left(\mathbb{F}_{q}\right)$. Also suppose that $\pi$ is optimal and $\mathfrak{m}$ is square-free. Then

$$
\operatorname{deg}(\pi)<q^{18 g+4 \operatorname{deg}(\infty)+1} \cdot q^{2 \operatorname{deg}(\mathfrak{m})} \cdot \operatorname{deg}(\mathfrak{m})^{3} .
$$

The result above is an analogue of Frey's celebrated degree conjecture, formulated originally for strong Weil curves over $\mathbb{Q}$. Our result completes [Papikian 2007], where a conjecture is made that implies that the Manin constant is zero (at 
least when $F$ is the rational function field and $\infty$ is the point at infinity), and where under this assumption an inequality significantly stronger than that in Theorem 1.5 was derived. As we saw, this hypothesis does not hold in general. In fact it is quite reasonable to expect that $m(E)$ be $d\left(\frac{1}{12} \operatorname{deg}\left(\Delta_{E}\right)+g-1\right)$ when $E$ is minimal in its isogeny class (see Section 7 for the definition). This is reflected by the fact that the contribution of our estimate of the term $c(E)^{-2}$ to our bound on the degree of the modular parametrisation is significant - it is of size $O\left(q^{\operatorname{deg}(\mathfrak{m})}\right)$.

Contents. In Section 2 we give an explicit description of the Hecke eigenform and prove Proposition 1.2. In Section 3 we carefully work out in detail the analogue of the theory of modular symbols for function fields, something that is missing from the current literature. In Section 4, we use these results to derive lower and upper bounds on the Manin constant in terms of the $p$-adic valuation of coefficients of $L$-functions of $E$ twisted with tamely ramified abelian characters. In Section 5, our aim is to relate the Galois module structure of the second coherent cohomology of the structure sheaf of elliptic surfaces equipped with a group action respecting the elliptic fibration to $\epsilon$-constants of Galois representations of the function field of the base in a special case. We prove a mild equivariant extension of Katz's conjecture relating the Newton and Hodge polygons, and with its aid we derive Theorem 1.3 from our previous results in Section 6. In Section 7 we show that the isogeny class of $E$ contains an elliptic curve whose $j$-invariant is not a $p$-th power, and we then use this result and the Pesenti-Szpiro inequality to deduce a bound on $m(E)$ in terms of the degree of the conductor of $E$ in Theorem 1.3. In Section 8 we first review [Ulmer 2002], and then use it and a classical result of Stickelberger on $p$-adic valuations of Gauss sums to prove Theorem 1.4. We show that the usual characterisation of strong Weil curves and optimal modular parametrisations holds in the function field setting as well in Section 9. In Section 10 we first show that a certain homomorphism defined in [Gekeler and Reversat 1996] has finite cokernel of exponent dividing $q^{\operatorname{deg}(\infty)}-1$. Then we combine this result with the bound in Section 7 and the work of Papikian to show Theorem 1.5.

\section{The normalised Hecke eigenform}

Notation 2.1. Let $\mathcal{O}$ denote the maximal compact subring of the ring $\mathbb{A}$ of adèles of $F$. Let $|\mathscr{C}|$ denote the set of closed points of $\mathscr{C}$. For every adèle $a \in \mathbb{A}$ and $x \in|\mathscr{C}|$, let $a_{x}$ denote the $x$-th component of $a$. Let $\mu, \mu^{*}$ be Haar measures on the locally compact abelian topological groups $\mathbb{A}$ and $\mathbb{A}^{*}$. Also assume that $\mu(0)$ and $\mu^{*}\left(O^{*}\right)$ are both equal to 1 . Since these measures are left-invariant with respect to the discrete subgroups $F^{*}$ and $F$ by definition, each induces a measure, on $F^{*} \backslash \mathbb{A}^{*}$ and $F \backslash \mathbb{A}$, respectively, both denoted by the same letter by abuse of notation. For every divisor $\mathfrak{m}$ on $\mathscr{C}$, let $\mathfrak{m} \mathfrak{O}, \mathbb{K}_{0}(\mathfrak{m})$ denote the sub- $\mathfrak{O}$-module of $\mathbb{A}$ generated by 
those idèles whose divisor is $\mathfrak{m}$, and the Hecke congruence subgroup of $\mathrm{GL}_{2}(\mathbb{A})$ of level $\mathfrak{m}$ :

$$
\mathbb{K}_{0}(\mathfrak{m})=\left\{\left(\begin{array}{ll}
a & b \\
c & d
\end{array}\right) \in \mathrm{GL}_{2}(\mathcal{O}) \mid c \in \mathfrak{m} \mathfrak{O}\right\}
$$

Let $\tau: F \backslash \mathbb{A} \rightarrow \mathbb{C}^{*}$ be a nontrivial continuous additive character. The composition of the quotient map $\mathbb{A} \rightarrow F \backslash \mathbb{A}$ and $\tau$ will be denoted by the same symbol by the usual abuse of notation. Let $\mathscr{D}$ denote the $\mathscr{O}$-module $\mathscr{D}=\{x \in \mathbb{A} \mid \tau(x \mathscr{O})=1\}$, and let $\mathfrak{d}$ be a divisor on $\mathscr{C}$ such that $\mathscr{D}=\mathfrak{d} \mathscr{O}$. Let $\mathfrak{n}$ be the conductor of $E$. The latter is an effective divisor on $\mathscr{C}$. We may assume that the divisor $\mathfrak{d}$ is relatively prime to $\mathfrak{n}$ by changing $\tau$, if necessary. Let $B$ denote the group scheme of invertible upper triangular two-by-two matrices. Let $P$ denote the group scheme of invertible upper triangular two-by-two matrices with 1 on the lower right corner. Finally let $Z$ denote the centre of the group scheme $\mathrm{GL}_{2}$.

Definition 2.2. For every idèle $u \in \mathbb{A}^{*}$, let $(u)$ denote the corresponding divisor on $\mathscr{C}$. Often we will denote $(u)$ simply by $u$ by slight abuse of notation, when this does not cause confusion. We will call two divisors $\mathfrak{m}$ and $\mathfrak{n}$ on $\mathscr{C}$ relatively prime if their support is disjoint. Let $\operatorname{Div}(\mathscr{C})$ denote the group of divisors on $\mathscr{C}$. We will call a function $f: \operatorname{Div}(\mathscr{C}) \rightarrow \mathbb{C}$ multiplicative if it vanishes on noneffective divisors, if $f(1)=1$, and if for every pair of relatively prime divisors $\mathfrak{n}$ and $\mathfrak{m}$ we have $f(\mathfrak{n m})=f(\mathfrak{n}) f(\mathfrak{m})$. Let $E$ be a nonisotrivial elliptic curve defined over $\mathscr{C}$ of conductor $\mathfrak{n}$. For every divisor $\mathfrak{r}$ on $\mathscr{b}$, let $\operatorname{deg}(\mathfrak{r})$ denote the degree of $\mathfrak{r}$. For every $x \in|\mathscr{G}|$, let $L_{x}(E, t)$ denote the local factor of the Hasse-Weil $L$-function of $E$ at $x$. It can be written as

$$
L_{x}(E, t)=\sum_{n=0}^{\infty} a\left(x^{n}\right)(t q)^{n \operatorname{deg}(x)} \in \mathbb{Z} \llbracket t \rrbracket
$$

for some $a\left(x^{n}\right) \in \mathbb{Z}\left[\frac{1}{p}\right]$. Let $a$ denote the unique multiplicative function into the multiplicative semigroup of $\mathbb{Q}$ such that $a\left(x^{n}\right)$ is the same as above for each natural number $n$ and each $x \in|\mathscr{C}|$. A continuous function $\psi_{E}: \mathrm{GL}_{2}(\mathbb{A}) \rightarrow \mathbb{Q}$ is called a normalised Hecke eigenform attached to $E$ is if it satisfies the following properties:

- It is automorphic: $\psi_{E}(\gamma h)=\psi_{E}(h)$ for all $\gamma \in \mathrm{GL}_{2}(F)$.

- It has trivial central character: $\psi_{E}(h z)=\psi_{E}(h)$ for all $z \in Z(\mathbb{A})$.

- It is right $\mathbb{K}_{0}(\mathfrak{n})$-invariant: $\psi_{E}(h k)=\psi_{E}(h)$ for all $k \in \mathbb{K}_{0}(\mathfrak{n})$.

- It is cuspidal:

$$
\int_{F \backslash \mathbb{A}} \psi_{E}\left(\left(\begin{array}{ll}
1 & x \\
0 & 1
\end{array}\right) h\right) d \mu(x)=0 \text { for all } h \in \mathrm{GL}_{2}(\mathbb{A}) .
$$


- Its Fourier coefficients are $a$ :

$a(\mathfrak{m} \mathfrak{d})=\mu(F \backslash \mathbb{A})^{-1} \int_{F \backslash \mathbb{A}} \psi_{E}\left(\left(\begin{array}{cc}\overline{\mathfrak{m}} & x \\ 0 & 1\end{array}\right)\right) \tau(-x) d \mu(x)$ for all $\mathfrak{m} \in \operatorname{Div}(\mathscr{b})$, where $\overline{\mathfrak{m}} \in \mathbb{A}^{*}$ and $(\overline{\mathfrak{m}})=\mathfrak{m}$.

Note that the last two conditions make sense because of the first; we may (and will) consider $\psi_{E}$ as a function on $\mathrm{GL}_{2}(F) \backslash \mathrm{GL}_{2}(\mathbb{A})$ as well.

Proposition 2.3. There is a unique normalised Hecke eigenform attached to E.

This claim is certainly very well known, and the only fact that needs an additional argument is that $\psi_{E}$ takes only rational values. Since we will shortly prove a stronger claim, we omit the proof. By a classical theorem of Harder [1974], the normalised Hecke eigenform $\psi_{E}$ is supported on a finite set as a function on the double coset $\mathrm{GL}_{2}(F) \backslash \mathrm{GL}_{2}(\mathbb{A}) / \mathbb{K}_{0}(\mathfrak{n}) Z(\mathbb{A})$. Let $L(E) \subseteq \mathbb{Q}$ denote the $\mathbb{Z}$-module generated by the values of $\psi_{E}$. Then there is a unique positive rational number $c(E) \in \mathbb{Q}$ such that $L(E)=c(E) \mathbb{Z}$.

Proposition 2.4. There is a nonnegative natural number $m(E)$ such that $c(E)=$ $p^{-m(E)}$.

As mentioned in the introduction, we call $m(E)$ the Manin constant of the elliptic curve $E$.

Proof. First we show that $L(E) \subseteq \mathbb{Z}\left[\frac{1}{p}\right]$. By the approximation theorem, we have $\mathrm{GL}_{2}(\mathbb{A})=\mathrm{GL}_{2}(F) P(\mathbb{A}) Z(\mathbb{A}) \mathbb{K}_{0}(\mathfrak{n})$. Therefore it will suffice to prove that $\psi_{E}(h) \in$ $\mathbb{Z}\left[\frac{1}{p}\right]$ for every element $h$ of $P(\mathbb{A})$. By the definition of Fourier coefficients,

$$
\psi_{E}\left(\left(\begin{array}{ll}
y & x \\
0 & 1
\end{array}\right)\right)=\sum_{\eta \in F^{*}} a\left(\eta y \mathfrak{d}^{-1}\right) \tau(\eta x)=\sum_{\eta \in S} a\left(\eta y \mathfrak{d}^{-1}\right)\left(\sum_{\epsilon \in \mathbb{F}_{q}^{*}} \tau(\eta \epsilon x)\right)
$$

for all $y \in \mathbb{A}^{*}, x \in \mathbb{A}$, where $S$ is a set of representatives of the quotient $\mathbb{F}_{q}^{*} \backslash F^{*}$, since $\psi_{E}$ is cuspidal. The character sums on the right side are all equal to -1 or $q-1$. Moreover, the sum above is finite. As $a(\mathfrak{m}) \in \mathbb{Z}\left[\frac{1}{p}\right]$ for every effective divisor $\mathfrak{m}$, the claim is now clear. Now we only need to show that $-1 \in L(E)$ in order to prove the proposition. Let $\overline{\mathfrak{d}} \in \mathbb{A}^{*}$ be such that $(\overline{\mathfrak{d}})=\mathfrak{d}$. Then by the Fourier expansion,

$$
\psi_{E}\left(\left(\begin{array}{ll}
\overline{\mathfrak{d}} & x \\
0 & 1
\end{array}\right)\right)=\sum_{\eta \in F^{*}} a(\eta) \tau(\eta x)=\sum_{\epsilon \in \mathbb{F}_{q}^{*}} a(\epsilon) \tau(\epsilon x)=\sum_{\epsilon \in \mathbb{F}_{q}^{*}} \tau(\epsilon x),
$$

because $F^{*} \cap \mathbb{O}=\mathbb{F}_{q}^{*}$ and $a(1)=1$ by definition. The character sum on the right side is equal to -1 if $x \notin \mathscr{D}$. 


\section{Epsilon constants and toric integrals}

Notation 3.1. For the rest of the paper, fix a prime number $l$ different from $p$. By the axiom of choice we may pick an isomorphism $l: \overline{\mathbb{Q}}_{l} \rightarrow \mathbb{C}$. We will identify $\overline{\mathbb{Q}}_{l}$ with $\mathbb{C}$ via $l$ in all that follows. Let $E_{l}$ be a finite extension of $\mathbb{Q}_{l}$ and let $\rho: \operatorname{Gal}(\bar{F} \mid F) \rightarrow \mathrm{GL}_{E_{l}}(W)$ be an $l$-adic representation on a finite dimensional vector space $W$ over $E_{l}$. Moreover, let $L(\rho, t) \in E_{l} \llbracket t \rrbracket$ denote the Grothendieck $L$-function associated to $\rho$ as defined in [Deligne 1973, 9.1]. By a classical theorem of Grothendieck [Deligne 1973, $\S 10$ ], the series $L(\rho, t)$ is a rational function in the variable $t$ and also satisfies the functional equation

$$
L(\rho, t)=\epsilon(\rho) t^{\alpha(\rho)} L\left(\rho^{\vee}, q^{-1} t^{-1}\right),
$$

where $\alpha(\rho) \in \mathbb{Z}, \epsilon(\rho) \in E_{l}^{*}$ and $\rho^{\vee}$ are the degree of the conductor of $\rho$ (in the sense of [Laumon 1987, page 179]), the $\epsilon$-constant of $\rho$, and the dual $l$-adic representation on $\operatorname{Hom}\left(W, E_{l}\right)$, respectively.

Notation 3.2. Let $K$ be a local field, let $d x$ be a Haar measure on $K$, and let $\psi$ be a nontrivial additive character on $K$. For every continuous homomorphism $\alpha: K^{*} \rightarrow \mathbb{C}^{*}$, let $\epsilon(K, \alpha, \psi, d x)$ denote the local $\epsilon$-factor attached to the triple $(\alpha, \psi, d x)$ as defined in [Deligne 1973, 3.3]. Let $W(\bar{K} \mid K)<\operatorname{Gal}(\bar{K} \mid K)$ denote the Weil group of $K$ (as defined in [Deligne 1973, 2.2.4]). Local class field theory furnishes an isomorphism $j: K^{*} \rightarrow W(\bar{K} \mid K)^{a b}$. We normalise this isomorphism so that for every uniformiser $\pi \in K^{*}$, the image of $j(\pi)$ with respect to the map $W(\bar{K} \mid K) \rightarrow \mathbb{Z}$ introduced in [Deligne 1973, 2.2.4] is the geometric Frobenius (similarly to [Deligne 1973, 2.3]). For every homomorphism $\alpha: \operatorname{Gal}(\bar{K} \mid K) \rightarrow$ $\mathbb{C}^{*}$, let the same symbol $\alpha$ denote the composition of $j$, considered here as an imbedding $j: K^{*} \rightarrow \operatorname{Gal}(\bar{K} \mid K)^{a b}$, with the map $\operatorname{Gal}(\bar{K} \mid K)^{a b} \rightarrow \mathbb{C}^{*}$ induced by the character $\alpha$ by slight abuse of notation.

Notation 3.3. For every $x \in|\mathscr{C}|$, let $F_{x}$ and $\mathscr{O}_{x}$ denote the completion of $F$ at $x$ and the valuation ring of $F_{x}$, respectively. For every $x \in|\mathscr{b}|$, let $\mu_{x}$ and $\mu_{x}^{*}$ be the unique Haar measures on the locally compact abelian topological groups $F_{x}$ and $F_{x}^{*}$, respectively, so that $\mu\left(O_{x}\right)$ and $\mu^{*}\left(0_{x}^{*}\right)$ are both equal to 1 . Moreover, for every $x \in|\mathscr{C}|$ let $\tau_{x}: F_{x} \rightarrow \mathbb{C}^{*}$ be the unique continuous additive character such that $\tau(a)=\prod_{x \in|\mathscr{G}|} \tau_{x}\left(a_{x}\right)$ for every $a \in \mathbb{A}$. For every homomorphism $\alpha: \operatorname{Gal}(\bar{F} \mid F) \rightarrow$ $\mathbb{C}^{*}$ and for every $x \in|\mathscr{\ell}|$, let $\alpha_{x}: \operatorname{Gal}\left(\bar{F}_{x} \mid F\right) \rightarrow \mathbb{C}^{*}$ denote the restriction of $\alpha$ onto the decomposition group at $x$.

Theorem 3.4. For every continuous homomorphism $\alpha: \operatorname{Gal}(\bar{K} \mid K) \rightarrow \mathbb{C}^{*}$ with finite image,

$$
\epsilon(\alpha)=q^{1-g} \prod_{x \in|\mathscr{6}|} \epsilon\left(F_{x}, \alpha_{x}, \tau_{x}, \mu_{x}\right)
$$


Proof. This is just a special case of [Laumon 1987, théorème 3.2.1.1].

Definition 3.5. For every divisor $\mathfrak{m}$ on $\mathscr{b}$, let $\underline{\mathfrak{m}}$ denote the support of $\mathfrak{m}$. Let $c \in \mathbb{A}^{*}$ be an idèle so that $\mathfrak{c}=(c)$ is an effective divisor on $\mathscr{b}$. For every such $c$, let $\hat{c} \in \mathbb{A}$ be the unique adèle such that $\hat{c}_{x}=c_{x}^{-1}$ for every $x \in \underline{\mathfrak{c}}$, and $\hat{c}_{x}=0$ otherwise. Let $\alpha: F \backslash \mathbb{A}^{*} \rightarrow \mathbb{C}^{*}$ be a continuous character with finite image whose conductor $\mathfrak{c}^{\prime}$ divides $\mathfrak{c}$. For every $z \in \mathbb{C}$, let $I\left(\psi_{E}, \mathfrak{c}, \alpha, z\right)$ denote the integral

$$
I\left(\psi_{E}, \mathfrak{c}, \alpha, z\right)=\int_{F^{*} \backslash \mathbb{A}^{*}} \psi_{E}\left(\left(\begin{array}{cc}
y & y \hat{c} \\
0 & 1
\end{array}\right)\right) \alpha(y) z^{\operatorname{deg}(y)} d \mu^{*}(y) \in \mathbb{C} .
$$

Lemma 3.6. The integral $I\left(\psi_{E}, \mathfrak{c}, \alpha, z\right)$ is well-defined and independent of the choice of $c$, as the notation indicates.

Proof. By [Tan 1993, Lemma 2], the integrand of $I\left(\psi_{E}, \mathfrak{c}, \alpha, z\right)$ is compactly supported. Hence $I\left(\psi_{E}, \mathfrak{c}, \alpha, z\right)$ is well-defined. Choose another idèle $c^{\prime} \in \mathbb{A}^{*}$ such that $\left(c^{\prime}\right)=\mathfrak{c}$. Then there is an $u \in \mathbb{O}^{*}$ such that $c^{\prime}=u c$ and therefore $\hat{c}^{\prime}=u \hat{c}$. Now the claim follows from the $\mathrm{GL}_{2}(\mathrm{O})$-invariance of $\psi_{E}$.

Notation 3.7. Let $W(\bar{F} \mid F)<\operatorname{Gal}(\bar{F} \mid F)$ denote the Weil group of $F$ (as defined in [Deligne 1973, 2.4]). Global class field theory furnishes an isomorphism

$$
\mathbf{j}: F^{*} \backslash \mathbb{A}^{*} \rightarrow W(\bar{F} \mid F)^{a b}
$$

that is compatible with the isomorphism between $F_{x}^{*}$ and $W\left(\bar{F}_{x} \mid F_{x}\right)^{a b}$ introduced in Notation 3.2 for every $x \in|\mathscr{C}|$ (in the sense of [Deligne 1973, 2.4]). For every homomorphism $\alpha: \operatorname{Gal}(\bar{F} \mid F) \rightarrow \mathbb{C}^{*}$, let the same symbol $\alpha$ denote the composition of $\mathbf{j}$, considered here as an imbedding $\mathbf{j}: F^{*} \backslash \mathbb{A}^{*} \rightarrow \operatorname{Gal}(\bar{F} \mid F)^{a b}$, with the map $\operatorname{Gal}(\bar{F} \mid F)^{a b} \rightarrow \mathbb{C}^{*}$ induced by the character $\alpha$. Moreover, let $\alpha$ also denote the composition of the quotient map $\mathbb{A}^{*} \rightarrow F^{*} \backslash \mathbb{A}^{*}$ and the map $\alpha: F^{*} \backslash \mathbb{A}^{*} \rightarrow \mathbb{C}^{*}$ introduced above by the usual abuse of notation.

Notation 3.8. For every divisor $\mathfrak{m}$ on $\mathscr{b}$ relatively prime to $\mathfrak{c}$, and for every $\overline{\mathfrak{m}} \in \mathbb{A}^{*}$ such that $(\overline{\mathfrak{m}})=\mathfrak{m}$, the complex number $\alpha(\overline{\mathfrak{m}})$ is independent of the choice of $\overline{\mathfrak{m}}$. We let $\alpha(\mathfrak{m})$ denote this common value. Let $\sigma_{E}$ denote the natural $l$-adic representation of $\operatorname{Gal}(\bar{F} \mid F)$ on the cohomology group $H^{1}\left(E_{\bar{F}}, \mathbb{Q}_{l}\right)$. By definition, $L(E, t)=L\left(\sigma_{E}, t\right)$. The twisted $L$-function $L\left(\sigma_{E} \otimes \alpha, t\right)$ is actually a polynomial in the variable $t$, and therefore it can be evaluated at any complex number $t=z$. Finally, let $G(E, \alpha, \mathfrak{c}, t) \in \mathbb{C}[t]$ denote the polynomial

$$
G(E, \alpha, \mathfrak{c}, t)=\prod_{x \in \underline{\mathfrak{c}}-\underline{\mathfrak{c}^{\prime}}}\left(-1+a(x) \alpha(x)(q t)^{\operatorname{deg}(x)}-\alpha(x)^{2} t^{2 \operatorname{deg}(x)}\right) .
$$


Proposition 3.9. Assume that $\mathfrak{c}$ is square-free and relatively prime to $\mathfrak{d n}$. Then

$$
\begin{aligned}
& I\left(\psi_{E}, \mathfrak{c}, \alpha, z\right) \\
& \quad=\alpha\left(\mathfrak{d}^{2} \mathfrak{c}^{\prime}\right) \epsilon\left(\alpha^{-1}\right) \prod_{x \in \mathfrak{c}}\left(q^{\operatorname{deg}(x)}-1\right)^{-1}\left(\frac{z^{2}}{q}\right)^{g-1} G(E, \alpha, \mathfrak{c}, z) L\left(\sigma_{E} \otimes \alpha, z q^{-1}\right) .
\end{aligned}
$$

Proof. According to the Fourier expansion of $\psi_{E}$, we have

$$
\psi_{E}\left(\left(\begin{array}{cc}
y & y \hat{c} \\
0 & 1
\end{array}\right)\right)=\sum_{\eta \in F^{*}} a\left(\eta y \mathfrak{d}^{-1}\right) \tau(\eta y \hat{c})
$$

for every $y \in \mathbb{A}^{*}$, and the sum on the right is finite. If we interchange this summation and the integration, we get

$$
I\left(\psi_{E}, \mathfrak{c}, \alpha, q^{-s}\right)=\int_{\mathbb{A}^{*}} a\left(y \mathfrak{d}^{-1}\right) \tau(y \hat{c}) \alpha(y) q^{-s \operatorname{deg}(y)} d \mu^{*}(y)
$$

for every $s \in \mathbb{C}$. This computation is justified by Lebesgue's convergence theorem if the second integral is absolutely convergent. This is so if $\operatorname{Re} s>\frac{1}{2}$, as the function $y \mapsto a\left(y \mathfrak{d}^{-1}\right)$ has support on $\mathfrak{d}^{-1} \mathfrak{O}$ and

$$
\left|a\left(y \mathfrak{d}^{-1}\right) \tau(y \hat{c})\right|=\left|a\left(y \mathfrak{d}^{-1}\right)\right| \leq 2 q^{-1 / 2 \operatorname{deg}\left(y \mathfrak{d}^{-1}\right)}
$$

by the Weil conjectures.

Let $\mathbb{A}_{\mathfrak{c}}$ and $\mathcal{O}_{\mathfrak{c}}$ denote the restricted direct products $\prod_{x \notin \mathfrak{c}}^{\prime} F_{x}$ and $\prod_{x \notin \mathfrak{c}}^{\prime} O_{x}$, respectively. Then $\mathbb{A}_{\mathfrak{c}}$ is a locally compact topological ring and $O_{\mathfrak{c}}$ is its maximal compact subring. Let $v_{\mathfrak{c}}^{*}$ be a Haar measure on $\mathbb{A}_{\mathfrak{c}}^{*}$ such that $v_{\mathfrak{c}}^{*}\left(\mathbb{O}_{\mathfrak{c}}^{*}\right)$ is equal to 1 . Let $|\cdot|_{x}$ be the absolute value on $F_{x}$ normalised so that $\mu_{x}(t \mathcal{O})=|t|_{x}$ for every $y \in F_{x}$. Using Fubini's theorem, the integral (1) can be rewritten as

$$
\int_{\mathbb{A}_{\mathfrak{c}}^{*}} a\left(y \mathfrak{d}^{-1}\right) \alpha(y) q^{-s \operatorname{deg}(y)} d \nu_{\mathfrak{c}}^{*}(y) \cdot \prod_{x \in \underline{\mathfrak{c}}} \int_{F_{x}^{*}} a(t) \tau_{x}\left(t c_{x}^{-1}\right) \alpha_{x}(t)|t|_{x}^{s} d \mu_{x}^{*}(t) .
$$

The integrand of the first integral of (2) is invariant under multiplication by $\mathbb{O}_{\mathfrak{c}}^{*}$. Therefore it is equal to

$$
\sum_{\substack{\mathfrak{m} \in \operatorname{Div}(\mathscr{C}) \\ \mathfrak{m} \cap \underline{\mathfrak{c}}=\varnothing}} a(\mathfrak{m}) \alpha(\mathfrak{m} \mathfrak{d}) q^{-s \operatorname{deg}(\mathfrak{m} \mathfrak{d})}=\alpha(\mathfrak{d}) q^{-s \operatorname{deg}(\mathfrak{d})} \cdot \prod_{x \notin \mathfrak{c}} L_{x}\left(\sigma_{E} \otimes \alpha, q^{-(s+1)}\right) .
$$

For every $x \in \underline{\mathfrak{c}}$, the corresponding term in the product (2) can be rewritten as

$$
\begin{aligned}
\int_{F_{x}^{*}} a(t) \tau_{x}\left(t c_{x}^{-1}\right) \alpha_{x}(t)|t|_{x}^{s} d \mu_{x}^{*}(t) & \\
= & \sum_{n=0}^{\infty} a\left(x^{n}\right) \alpha_{x}\left(c_{x}\right)^{n}\left|c_{x}\right|_{x}^{n s} \int_{\mathbb{O}_{x}^{*}} \tau_{x}\left(t c_{x}^{n-1}\right) \alpha_{x}(t) d \mu_{x}^{*}(t),
\end{aligned}
$$


because $c_{x} \in F_{x}^{*}$ is a uniformiser, and $a\left(x^{n}\right)=0$ if $n<0$. Suppose now that $x$ divides the conductor of $\alpha$. Then the restriction of $\alpha_{x}$ onto $0_{x}^{*}$ is a nontrivial character, and therefore

$$
\int_{\mathcal{O}_{x}^{*}} \tau_{x}\left(t c_{x}^{n-1}\right) \alpha_{x}(t) d \mu_{x}^{*}(t)=\left\{\begin{array}{cl}
\frac{\alpha_{x}\left(c_{x}\right)}{q^{\operatorname{deg}(x)}-1} \epsilon\left(F_{x}, \alpha_{x}^{-1}, \tau_{x}, \mu_{x}\right) & \text { if } n=0, \\
0 & \text { otherwise, }
\end{array}\right.
$$

by [Deligne 1973, (3.4.3.2)] and the fact that the additive character $\tau_{x}$ restricted to $\mathrm{O}_{x}$ is trivial. Hence

$$
\int_{F_{x}^{*}} a(t) \tau_{x}\left(t c_{x}^{-1}\right) \alpha_{x}(t)|t|_{x}^{s} d \mu_{x}^{*}(t)=\frac{\alpha_{x}\left(c_{x}\right)}{q^{\operatorname{deg}(x)}-1} \epsilon\left(F_{x}, \alpha_{x}^{-1}, \tau_{x}, \mu_{x}\right)
$$

in this case. Otherwise, the restriction of $\alpha_{x}$ onto $0_{v}^{*}$ is the trivial character, and therefore the left side of (4) is equal to

$$
\frac{-1}{q^{\operatorname{deg}(x)}-1}+\sum_{n=1}^{\infty} a\left(x^{n}\right) \alpha_{x}\left(c_{x}\right)^{n}\left|c_{x}\right|_{x}^{n s}=L_{x}\left(\sigma_{E} \otimes \alpha, q^{-(s+1)}\right)-\frac{q^{\operatorname{deg}(x)}}{q^{\operatorname{deg}(x)}-1} .
$$

Because both $\sigma_{E}$ and $\alpha$ are unramified at $x$, we have

$$
L_{x}\left(\sigma_{E} \otimes \alpha, q^{-(s+1)}\right)=\left(1-a(x) \alpha(x) q^{-s \operatorname{deg}(x)}+\alpha(x)^{2} q^{-(2 s+1) \operatorname{deg}(x)}\right)^{-1} .
$$

Hence, in this case,

$$
\begin{aligned}
& \int_{F_{x}^{*}} a(t) \tau_{x}\left(t c_{x}^{-1}\right) \alpha_{x}(t)|t|_{x}^{s} d \mu_{x}^{*}(t) \\
&=\frac{1}{q^{\operatorname{deg}(x)}-1}\left(-1+a(x) \alpha(x) q^{(1-s) \operatorname{deg}(x)}-\alpha(x)^{2} q^{-2 s \operatorname{deg}(x)}\right) \\
& \cdot L_{x}\left(\sigma_{E} \otimes \alpha, q^{-(s+1)}\right) .
\end{aligned}
$$

By Theorem 3.4, we have

$$
\epsilon\left(\alpha^{-1}\right)=q^{1-g} \alpha^{-1}(\mathfrak{d}) q^{\operatorname{deg}(\mathfrak{d})} \prod_{x \in \underline{\mathfrak{c}}^{\prime}} \epsilon\left(F_{x}, \alpha_{x}^{-1}, \tau_{x}, \mu_{x}\right),
$$

because according to [Deligne 1973, (3.4.3.1)], we have

$$
\epsilon\left(F_{x}, \alpha_{x}^{-1}, \tau_{x}, \mu_{x}\right)=\alpha_{x}^{-1}\left(\mathfrak{d}_{x}\right) q^{\operatorname{deg}\left(\mathfrak{d}_{x}\right)}
$$

if $\alpha_{x}$ is unramified, since we assumed that $\mu_{x}\left(\mathbb{O}_{x}\right)=1$ and $\mathfrak{c}$ and $\mathfrak{d}$ are relatively prime. Combining (3), (6), (9), and (10), we get

$$
\begin{array}{r}
I\left(\psi_{E}, \mathfrak{c}, \alpha, q^{-s}\right)=\alpha\left(\mathfrak{d}^{2} \mathfrak{c}^{\prime}\right) \epsilon\left(\alpha^{-1}\right) q^{g-1-(s+1) \operatorname{deg}(\mathfrak{d})} \prod_{x \in \mathfrak{c}}\left(q^{\operatorname{deg}(x)}-1\right)^{-1} \\
\cdot G\left(E, \alpha, \mathfrak{c}, q^{-s}\right) L\left(\sigma_{E} \otimes \alpha, q^{-(s+1)}\right),
\end{array}
$$


if we also use that $L_{x}\left(\sigma_{E} \otimes \alpha, q^{-(s+1)}\right)=1$ when $x \in \underline{\mathfrak{c}}^{\prime}$. Because $\operatorname{deg}(\mathfrak{d})=2 g-2$, the claim now follows for every complex number $q^{-s}$ such that $\operatorname{Re} s>\frac{1}{2}$. But both sides of the equation in the proposition above are polynomials in $z$; hence the claim must hold for every complex number as well.

\section{Lower and upper bounds}

Notation 4.1. For every field $K$, let $\bar{K}$ denote a separable closure of $K$. Let $v_{q}$ : $\overline{\mathbb{Q}}_{p}^{*} \rightarrow \mathbb{Q}$ denote the $p$-adic valuation normalised such that $v_{q}(q)=1$. Every polynomial $P(t) \in \overline{\mathbb{Q}}_{p}[t]$ can be written in the form

$$
P(t)=a t^{k} \prod_{i=1}^{n-k}\left(1-\lambda_{i} t\right), \quad a \in \overline{\mathbb{Q}}_{p}^{*}, \quad \lambda_{i} \in \overline{\mathbb{Q}}_{p},
$$

where the $\lambda_{i}$ are the reciprocal roots of $P(t)$. Let $l_{q}(P(t)) \in \mathbb{Q}$ denote the nonnegative number

$$
l_{q}(P(t))=\sum_{v_{q}\left(\lambda_{i}\right) \leq 1}\left(1-v_{q}\left(\lambda_{i}\right)\right) .
$$

Let $\mu_{\infty}, \mu_{\infty, p} \subset \overline{\mathbb{Q}}_{p}^{*}$ denote the subgroup of roots of unity and of roots of unity whose order is prime to $p$, respectively.

Lemma 4.2. With the same notation as above,

$$
\min _{\epsilon \in \mu_{\infty}}\left(v_{q}\left(P\left(\epsilon q^{-1}\right)\right)\right)=v_{q}(a)-k-l_{q}(P(t)) .
$$

Moreover, the minimum is attained at all but finitely many $\epsilon \in \mu_{\infty, p}$.

Proof. For a fixed $i=1,2, \ldots, k$ and for every $\epsilon \in \mu_{\infty}$, we have

$$
v_{q}\left(1-\lambda_{i} \epsilon q^{-1}\right)=-1+v_{q}\left(\lambda_{i}-\epsilon^{-1} q\right) \geq \min \left\{0, v_{q}\left(\lambda_{i}\right)-1\right\},
$$

so for all but finitely many $\epsilon \in \mu_{\infty, p}$, we have

$$
v_{q}\left(1-\lambda_{i} \epsilon q^{-1}\right)=\min \left\{0, v_{q}\left(\lambda_{i}\right)-1\right\} .
$$

Therefore, for all but finitely many $\epsilon \in \mu_{\infty, p}$,

$$
v_{q}\left(P\left(\epsilon q^{-1}\right)\right)=\min _{\zeta \in \mu_{\infty}}\left(v_{q}\left(P\left(\zeta q^{-1}\right)\right)\right)=v_{q}(a)-k+\sum_{i=1}^{\operatorname{deg}(P)} \min \left\{0, v_{q}\left(\lambda_{i}\right)-1\right\} .
$$

Let $\mathfrak{c}$ be a square-free effective divisor on $\mathscr{C}$ that is relatively prime to $\mathfrak{d} n$. Let $\alpha: F \backslash \mathbb{A}^{*} \rightarrow \mathbb{C}^{*}$ be a continuous character with finite image whose conductor $\mathfrak{c}^{\prime}$ divides $\mathfrak{c}$.

Lemma 4.3. The minimum of $v_{q}(G(E, \alpha, \mathfrak{c}, \epsilon))$ for $\epsilon$ ranging over $\mu_{\infty}$ is zero, and it is attained at all but finitely many $\epsilon \in \mu_{\infty, p}$. 
Proof. For every $x \in \underline{\mathfrak{c}}-\underline{\mathfrak{c}}^{\prime}$, we have $q^{\operatorname{deg}(x)} a(x) \in \mathbb{Z}$. Hence, for every $\epsilon \in \mu_{\infty}$ and $x$ as above, we have

$$
v_{q}\left(-1+a(x) \alpha(x) q^{\operatorname{deg}(x)} \epsilon^{\operatorname{deg}(x)}-\alpha(x)^{2} \epsilon^{2 \operatorname{deg}(x)}\right) \geq 0,
$$

and for all but finitely many $\epsilon \in \mu_{\infty, p}$, the left side is equal to 0 . The claim follows by taking the product over all $x \in \underline{\mathfrak{c}}-\underline{\mathfrak{c}}^{\prime}$.

Lemma 4.4. For every $y \in \mathbb{A}^{*}$ and $x \in \mathbb{A}$, there are $\eta \in F, u \in \mathbb{O}$ and $c \in \mathbb{A}^{*}$ such that (c) is a square-free effective divisor that is relatively prime to $\mathfrak{n}$ and

$$
x+u+y^{-1} \eta=\hat{c} .
$$

Proof. Let $z \in \mathbb{A}^{*}$ be the unique idèle such that for every $v \in|\mathscr{C}|$ we have $z_{v}=x_{v}^{-1}$ if $x_{v} \notin \mathfrak{O}_{v}$, and $z_{v}=1$ otherwise. Then $\mathfrak{z}=(z)$ is an effective divisor. Let $\mathfrak{y}$ denote the divisor of $y^{-1}$ and let $\mathfrak{b}$ be a square-free effective divisor whose degree is at least $2 g-1-\operatorname{deg}(\mathfrak{y})$ and which is relatively prime to $\mathfrak{n} \mathfrak{z}$. Let $Z$ denote the closed scheme of $\mathscr{C}$ whose sheaf of ideals is $\mathrm{O}_{\mathscr{C}}(\mathfrak{z})^{\vee} \subseteq \mathrm{O}_{\mathscr{C}}$, where for every vector bundle $\mathscr{F}_{F}$ on $\mathscr{C}$ we let $\mathscr{F}^{\vee}$ denote the dual of $\mathscr{F}$. We have an exact sequence

$$
H^{0}\left(\mathfrak{b}, \mathcal{O}_{\mathscr{C}}(\mathfrak{y} \mathfrak{b})\right) \longrightarrow H^{0}\left(\mathscr{b}, \mathcal{O}_{\mathscr{C}}(\mathfrak{z} \mathfrak{y} \mathfrak{b})\right) \stackrel{i_{Z}}{\longrightarrow} H^{0}\left(Z,\left.\mathcal{O}_{\mathscr{C}}(\mathfrak{z} \mathfrak{y} \mathfrak{b})\right|_{Z}\right),
$$

where the first map is induced by the inclusion $O_{\mathscr{C}}(\mathfrak{y} \mathfrak{b}) \subset O_{\mathscr{C}}(\mathfrak{z} \mathfrak{y} \mathfrak{b})$, and the second map $i_{Z}$ is the restriction map. By the Riemann-Roch theorem for curves,

$$
\operatorname{dim}_{\mathbb{F}_{q}} H^{0}(\mathscr{C}, \mathscr{F})=2-2 g+\operatorname{deg}(\mathscr{F})
$$

for every line bundle $\mathscr{F}$ on $\mathscr{C}$ whose degree is at least $2 g-1$. Comparing the dimensions of $H^{0}\left(\mathfrak{C}_{,} \mathcal{O}_{\mathfrak{C}}(\mathfrak{y} \mathfrak{b})\right)$ and $H^{0}\left(\mathfrak{C}_{,}, \mathrm{O}_{\mathfrak{C}}(\mathfrak{z} \mathfrak{y} \mathfrak{b})\right)$, we get that the image of $i_{Z}$ has dimension $\operatorname{deg}(\mathfrak{z})$ over $\mathbb{F}_{q}$. But the dimension of $H^{0}\left(Z,\left.\mathscr{O}_{\mathfrak{b}}(\mathfrak{z} \mathfrak{y} \mathfrak{b})\right|_{Z}\right)$ is the same, and hence $i_{Z}$ is surjective.

Let $b \in \mathbb{A}^{*}$ be an idèle such that $(b)=\mathfrak{b}$ and $b_{v}=1$ for every $v \notin \underline{\mathfrak{b}}$. Recall that for every idèle $t \in \mathbb{A}^{*}$ we have: $H^{0}\left(\mathscr{C}, \mathcal{O}_{\mathscr{C}}(t)\right)=F \cap t^{-1} \mathcal{O}$. Moreover, for every such $t$ we have $H^{0}\left(Z,\left.O_{\mathscr{C}}(t z)\right|_{Z}\right)=(t z)^{-1} \mathbb{O} / t^{-1} \mathcal{O}$. Under these identifications, $i_{Z}$ is the composition of the inclusion $F \cap(z b)^{-1} y \mathcal{O} \rightarrow(z b)^{-1} y \mathcal{O}$ and the reduction map $(z b)^{-1} y \mathcal{O} \rightarrow(z b)^{-1} y \mathcal{O} / b^{-1} y \mathcal{O}$. Therefore, there is an $\eta \in F$ such that for every $v \in|\mathscr{C}|$, we have $\left(z b y^{-1} \eta\right)_{v} \in-1+z_{v} \mathbb{O}_{v}$ if $v \in \underline{\mathfrak{z}}$, and $\left(z y^{-1} b \eta\right)_{v}=\left(y^{-1} b \eta\right)_{v} \in \mathbb{O}_{v}$ otherwise. Because $\mathfrak{b}$ is relatively prime to $\mathfrak{z}$, we get that $\left(y^{-1} \eta\right)_{v} \in-x_{v}+\mathbb{O}_{v}$ if $v \in \underline{\mathfrak{z}}$. Let $c \in \mathbb{A}^{*}$ be the unique idèle such that $c_{v}=\left(y \eta^{-1}\right)_{v}$ if $v \notin \underline{\mathfrak{z}}$ and $\left(y^{-1} \eta\right)_{v} \notin \mathbb{O}_{v}$, and $c_{v}=1$ otherwise. Thus $(c)$ divides $\mathfrak{b}$, so it is a square-free effective divisor relatively prime to $\mathfrak{n}$. Let $u=-x-y^{-1} \eta+\hat{c}$. By the above, $u \in \mathbb{O}$, so this choice of $\eta, c$ and $u$ satisfies the requirements of the claim.

Let $\mathbb{X}(\mathfrak{n})$ denote the set of tamely ramified continuous characters $F \backslash \mathbb{A}^{*} \rightarrow \mathbb{C}^{*}$ with finite image whose conductor is relatively prime to $\mathfrak{n}$. 


\section{Proposition 4.5.}

$$
m(E) \geq d \cdot \sup _{\alpha \in \mathbb{X}(\mathfrak{n})}\left(l_{q}\left(L\left(\sigma_{E} \otimes \alpha, t\right)\right)+g-1-v_{q}\left(\epsilon\left(\alpha^{-1}\right)\right)\right) .
$$

When $p$ does not divide the order of $\operatorname{Pic}_{0}(\mathscr{C})\left(\mathbb{F}_{q}\right)$, the two sides are equal.

Proof. Fix an isomorphism $\overline{\mathbb{Q}}_{p} \cong \mathbb{C}$ and let $\alpha \in \mathbb{X}(\mathfrak{n})$ have conductor $\mathfrak{c}$. Without loss of generality, we may assume that $\mathfrak{c}$ is relatively prime to $\mathfrak{d}$ by changing $\tau$, if necessary. According to Proposition 3.9,

$$
I\left(\psi_{E}, \mathfrak{c}, \alpha, z\right)=\alpha\left(\mathfrak{d}^{2} \mathfrak{c}^{\prime}\right) \epsilon\left(\alpha^{-1}\right) \prod_{x \in \mathfrak{c}}\left(q^{\operatorname{deg}(x)}-1\right)^{-1}\left(z^{2} / q\right)^{g-1} L\left(\sigma_{E} \otimes \alpha, z q^{-1}\right) .
$$

Let $O_{\mathfrak{c}}<\mathbb{A}^{*}$ denote the subgroup $\mathbb{O}^{*}$ if $\mathfrak{c}=1$, and $1+\mathfrak{c} O$ otherwise. The integrand of the integral on the left side of (12) is constant on the cosets of the subgroup $u_{\mathfrak{c}}<F^{*} \backslash \mathbb{A}^{*}$, where $u_{\mathfrak{c}}=\left(\mathbb{F}_{q}^{*} \cap O_{c}\right) \backslash 0_{c}$. Because $\mathfrak{c}$ is square-free, $p$ does not divide $\left|0^{*} / O_{\mathfrak{c}}\right|$. Hence $\mu^{*}\left(U_{\mathfrak{c}}\right)$ is a rational number whose denominator is not divisible by $p$. Therefore $I\left(\psi_{E}, \mathfrak{c}, \alpha, \epsilon\right) \in p^{-m(E)} \mathbb{Z}_{p}[\epsilon]$ for every $\epsilon \in \mu_{\infty}$. In particular, $v_{q}\left(I\left(\psi_{E}, \mathfrak{c}, \alpha, \epsilon\right)\right)$ is at least $-m(E) / d$. Note that for every $\alpha \in \mathbb{X}(\mathfrak{n})$,

$$
L\left(\sigma_{E} \otimes \alpha, t\right) \in 1+t \overline{\mathbb{Q}}_{p}[t],
$$

and hence by Lemma 4.2 there is an $\epsilon \in \mu_{\infty}$ such that $v_{q}\left(L\left(\sigma_{E} \otimes \alpha, \epsilon q^{-1}\right)\right)=$ $-l_{q}\left(L\left(\sigma_{E} \otimes \alpha, t\right)\right)$. Hence the first part of the claim above is true.

Assume now that $p$ does not divide the order of $\operatorname{Pic}_{0}(\mathscr{C})\left(\mathbb{F}_{q}\right)$, and let $h(E)$ denote the right side of the inequality in Proposition 4.5. As we already noted in the proof of Proposition 2.4, in order to show the second half of the claim, we only need to show that $p^{h(E)} \psi_{E}(g) \in \mathbb{Z}$ for every element $g=\left(\begin{array}{cc}y & y x \\ 0 & 1\end{array}\right) \in P(\mathbb{A})$. By Lemma 4.4, there are $\eta \in F, u \in \mathbb{O}$ and $c \in \mathbb{A}^{*}$ such that $(c)$ is a square-free effective divisor that is relatively prime to $\mathfrak{n}$ and $x+u+y^{-1} \eta=\hat{c}$. Because

$$
\left(\begin{array}{ll}
1 & \eta \\
0 & 1
\end{array}\right) \cdot\left(\begin{array}{cc}
y & y x \\
0 & 1
\end{array}\right) \cdot\left(\begin{array}{ll}
1 & u \\
0 & 1
\end{array}\right)=\left(\begin{array}{cc}
y & y\left(x+u+y^{-1} \eta\right) \\
0 & 1
\end{array}\right)
$$

and $\psi_{E}$ is invariant on the left with respect to $P(F)$ and on the right with respect to $P(\mathbb{O})$, we may assume that $x=\hat{c}$.

Let $\mathfrak{c}$ be $(c)$ and let $H$ denote the quotient group $\mathbb{A}^{*} / F^{*} \mathscr{O}_{\mathfrak{c}}$, where we continue to use the notation above. Then $H$ can be decomposed as a direct product $G \times \mathbb{Z}$, where $G$ is a finite subgroup. By class field theory, $|G|=\left|\mathrm{Pic}_{0}(\mathscr{C})\right| \cdot\left|\mathbb{O}^{*} /\left(\mathbb{O}_{\mathfrak{c}} \mathbb{F}_{q}^{*}\right)\right|$. As noted above, $p$ does not divide $\left|0^{*} / O_{\mathfrak{c}}\right|$, and hence does not divide the order of $G$ by our assumption. Let $(\cdot)_{H}: \mathbb{A}^{*} \rightarrow H$ be the quotient map and let $[\cdot]: H \rightarrow G$ denote the projection onto the factor $G$. Note that for every $y \in \mathbb{A}^{*}$, the value

$$
\psi_{E}\left(\left(\begin{array}{cc}
y & y \hat{c} \\
0 & 1
\end{array}\right)\right) t^{\operatorname{deg}(y)} \in \mathbb{Q}[G]\left[t, t^{-1}\right]
$$


only depends on $(y)_{H}$. Let $f: H \rightarrow \mathbb{Q}\left[t, t^{-1}\right]$ be the corresponding function, and define $I=\sum_{\substack{g \in G \\ n \in \mathbb{Z}}} c_{g, n} g t^{n} \in \mathbb{Q}[G]\left[t, t^{-1}\right]$ by the formula

$$
I=\sum_{g \in G}\left(\sum_{\substack{h \in H \\[h]=g}} f(h)\right) g .
$$

The function $I$ is well-defined because for every $g \in G$, the set of those $h \in H$ such that $[h]=g$ and $f(y) \neq 0$ is finite. Also the set $\left\{c_{g, n} \mid g \in G, n \in \mathbb{Z}\right\}$ and the image of the function $\mathbb{A}^{*} \rightarrow \mathbb{Q}$ given by the rule $y \mapsto \psi_{E}\left(\left(\begin{array}{cc}y & y \hat{c} \\ 0 & 1\end{array}\right)\right)$ are equal, and hence it will suffice to show that $p^{h(E)} I \in \mathbb{Z}[G]\left[t, t^{-1}\right]$. For every group homomorphism $\alpha: G \rightarrow \overline{\mathbb{Q}}_{p}^{*}$, by slight abuse of notation, let the same symbol $\alpha$ denote the unique ring homomorphism $\mathbb{Q}[G]\left[t, t^{-1}\right] \rightarrow \overline{\mathbb{Q}}_{p}\left[t, t^{-1}\right]$, whose restriction onto $G$ is $\alpha$ and $\alpha(t)=t$. Because $p$ does not divide $|G|$, it will suffice to show that $v_{q}(a) \leq$ $-h(E) / d$ for every coefficient $a$ of $\alpha(I)$ for every $\alpha$ as above. Let the symbol $\alpha$ denote also the composition $\alpha \circ[\cdot] \circ(\cdot)_{H}: \mathbb{A}^{*} \rightarrow \overline{\mathbb{Q}}_{p}^{*}$. Choose the character $\tau$ so that $\mathfrak{d}$ is relatively prime to $\mathfrak{c}$. Then $\alpha(I)$ is the polynomial

$$
\alpha(I)=\alpha\left(\mathfrak{d}^{2} \mathfrak{c}^{\prime}\right) \epsilon\left(\alpha^{-1}\right) \prod_{x \in \mathfrak{c}}\left(q^{\operatorname{deg}(x)}-1\right)^{-1}\left(\frac{t^{2}}{q}\right)^{g-1} G(E, \alpha, \mathfrak{c}, t) L\left(\sigma_{E} \otimes \alpha, t q^{-1}\right)
$$

by Proposition 3.9. Since for every polynomial $P(t)=\sum_{k=0}^{N} b_{k} t^{k} \in \overline{\mathbb{Q}}_{p}$ we have

$$
\min _{\epsilon \in \mu_{\infty}}\left(v_{q}(P(\epsilon))\right)=\min _{0 \leq k \leq N}\left(v_{q}\left(b_{k}\right)\right),
$$

the claim now follows from Lemmas 4.2 and 4.3.

\section{Galois module structure of the coherent cohomology of elliptic surfaces}

Definition 5.1. In this section, $G$ will be a finite group. Let $A$ be a noetherian ring and let $Y$ be a scheme that is separated and of finite type over $\operatorname{Spec}(A)$. By an $A[G]$-module on $Y$, we mean a sheaf of $A[G]$-modules on $Y$. These form the objects of a category whose morphisms are maps respecting the $A[G]$-module structure. Suppose now that $\mathscr{F}$ is an $A[G]$-module on $Y$ that is also an $\mathscr{O}_{Y}$-module in such a way that the actions of $O_{Y}$ and $G$ commute and the $A[G]$-module structure of $\mathscr{F}$ respects the structure morphism $Y \rightarrow \operatorname{Spec}(A)$. If $\mathscr{F}$ is also a quasicoherent or coherent $\mathrm{O}_{Y}$-module, then we will call $\mathscr{F}_{F}$ a quasicoherent or coherent $\mathrm{O}_{Y}[G]$ module, respectively. As noted in [Chinburg 1994, p. 447], there are enough injectives in the category of $A[G]$-modules on $Y$, and hence the global section functor $\Gamma$ has a derived functor into the localisation of the category of complexes of $A[G]$-complexes bounded from below with respect to the multiplicative system of quasiisomorphisms which will be denoted by $R \Gamma^{+}$. 
Definition 5.2. An $A[G]$-module $M$ is cohomologically trivial if the Tate cohomology group $\widehat{H}^{i}(H, M)$ vanishes for all subgroups $H$ of $G$ and for all integers $i$. Let $C T(A[G])$ denote the Grothendieck group of all finitely generated $A[G]$-modules that are cohomologically trivial. For every cohomologically trivial $A[G]$-module $M$, let $[M]$ denote its class in $C T(A[G]))$. Suppose that $\mathscr{F}$ is a quasicoherent $O_{Y}$-module such that each stalk of $\mathscr{F}$ is a cohomologically trivial $A[G]$-module. Then by [Chinburg 1994, Theorem 1.1], the complex $R \Gamma^{+}(\mathscr{F})$ is isomorphic in the derived category of $A[G]$-modules to a bounded complex $M^{*}$ of finitely generated cohomologically trivial $A[G]$-modules. Moreover, the Euler characteristic $\sum(-1)^{i}\left[M^{i}\right]$ in $C T(A[G])$ only depends on $\mathscr{F}$ and will be denoted by $\chi(\mathscr{F})$.

Definition 5.3. Let $X$ be a normal scheme that is of finite type over $\operatorname{Spec}(A)$. Assume that the finite group $G$ acts on $X$ on the left. Let $\mathscr{F}$ be a coherent sheaf on $X$. A $G$-linearisation on $\mathscr{F}$ is a collection $\Psi=\left\{\psi_{g}\right\}_{g \in G}$ of isomorphisms $\psi_{g}$ : $g_{*}(\mathscr{F}) \rightarrow \mathscr{F}$ for every $g \in G$ such that

- $\psi_{1}=\operatorname{Id}_{\mathscr{F}}$ and

- for every $g, h \in G$ we have $\psi_{h g}=\psi_{h} \circ h_{*}\left(\psi_{g}\right)$,

where $h_{*}\left(\psi_{g}\right):(h g)_{*}(\mathscr{F})=h_{*}\left(g_{*}(\mathscr{F})\right) \rightarrow h_{*}(\mathscr{F})$ is the direct image of the map $\psi_{g}: g_{*}(\mathscr{F}) \rightarrow \mathscr{F}$ under the action of $h$. We define a $G$-sheaf over $X$ to be a sheaf on $X$ equipped with a $G$-linearisation. A coherent $G$-sheaf is a coherent sheaf on $X$ equipped with a $G$-linearisation $\Psi$ such that $\psi_{g}: g_{*}(\mathscr{F}) \rightarrow \mathscr{F}$ is $\mathscr{O}_{X}$-linear for every $g \in G$.

Definition 5.4. Let $f: X \rightarrow Y$ be a tame $G$-cover as defined in [Chinburg 1994, Definition 2.2], and let $\mathscr{F}$ be a coherent $G$-sheaf on $X$. The $G$-linearisation on $\mathscr{F}$ induces an $O_{Y}$-linear action of $G$ on the direct image sheaf $f_{*}(\mathscr{F})$ that makes the latter a coherent $O_{Y}[G]$-module. By [Chinburg 1994, Theorem 2.7], each stalk of the $0_{Y}[G]$-sheaf $f_{*}(\mathscr{F})$ is a cohomologically trivial $A[G]$-module. Hence the Euler characteristic $\chi\left(f_{*}(\mathscr{F})\right) \in C T(A[G])$ introduced in Definition 5.2 is well-defined, and will be denoted by $\chi(G, \mathscr{F})$.

Now suppose that $A$ is a field and that its characteristic does not divide the order of $G$. Then every finitely generated $A[G]$-module is cohomologically trivial. Also assume that $Y$ is proper over $\operatorname{Spec}(A)$, and let $\mathscr{F}$ be again a coherent $G$-sheaf on $X$. Then for every $n \in \mathbb{N}$, the cohomology group $H^{n}(X, \mathscr{F})$ is a cohomologically trivial, finitely generated $A[G]$-module with respect to the natural $A[G]$-action.

Lemma 5.5. $\chi(G, \mathscr{F})=\sum_{n \in \mathbb{N}}(-1)^{n}\left[H^{n}(X, \mathscr{F})\right] \in C T(A[G])$.

Proof. Because finite maps are affine, the higher derived sheaves $R^{i} f_{*}(\mathscr{F})$ are vanishing. Hence $H^{n}(X, \mathscr{F})=H^{n}\left(Y, f_{*}(\mathscr{F})\right)$ as $A[G]$-modules. Now the claim follows from [Chinburg 1994, Proposition 1.5]. 
Suppose now that $f: X \rightarrow Y$ as above is a map of smooth, projective curves over $\operatorname{Spec}(A)$. Let $\mathscr{L}$ be a line bundle on $Y$. The line bundle $f^{*}(\mathscr{L})$ on $X$ is naturally equipped with the structure of a coherent $G$-sheaf.

Lemma 5.6. Keeping the same notation and assumptions, we have in $C T(A[G])$

$$
\chi\left(G, f^{*}(\mathscr{L})\right)=\chi\left(G, O_{X}\right)+\operatorname{deg}(\mathscr{L})[A[G]] .
$$

Proof. Of course we are going to show the claim with the usual dévissage argument. By the Riemann-Roch theorem, there is a divisor $D$ on $Y$ whose support is disjoint from the ramification divisor of the cover $f$ and $\mathscr{L}=O_{Y}(D)$. First assume that $D$ is effective. When $\operatorname{deg}(D)=0$ the claim is obvious. Otherwise $D=D^{\prime}+\mathfrak{p}$, where $D^{\prime}$ is an effective divisor with $\operatorname{deg}\left(D^{\prime}\right)<\operatorname{deg}(D)$ and $\mathfrak{p}$ is a closed point on $Y$. There is a short exact sequence

$$
0 \rightarrow f^{*}\left(\mathscr{O}_{Y}\left(D^{\prime}\right)\right) \rightarrow f^{*}\left(\mathscr{O}_{Y}(D)\right) \rightarrow f^{*}(A \mathfrak{p}) \rightarrow 0,
$$

where $A \mathfrak{p}$ denotes the skyscraper sheaf on $Y$ with support $\mathfrak{p}$. Because $\mathfrak{p}$ is not in the ramification locus of $f$, we have $H^{0}\left(f_{*} f^{*}(A \mathfrak{p}), Y\right) \cong A[G]^{\operatorname{deg}(\mathfrak{p})}$ as $A[G]$-modules. Moreover, all higher cohomology groups of the skyscraper sheaf $f_{*} f^{*}(A \mathfrak{p})$ vanish. Hence by the additivity of the Euler characteristic we get

$$
\chi\left(G, f^{*}\left(\mathscr{O}_{Y}(D)\right)\right)=\chi\left(G, f^{*}\left(\mathscr{O}_{Y}\left(D^{\prime}\right)\right)\right)+\operatorname{deg}(\mathfrak{p})[A[G]] .
$$

Now the claim follows by induction on $\operatorname{deg}(D)$. Consider next the general case and write $D=D_{1}-D_{2}$, where $D_{1}$ and $D_{2}$ are divisors on $X$ whose supports are disjoint. We are going to prove the claim by induction on $\operatorname{deg}\left(D_{2}\right)$. We already proved the claim when $\operatorname{deg}\left(D_{2}\right)=0$. Otherwise $D_{2}=D_{2}^{\prime}+\mathfrak{p}$, where $D_{2}^{\prime}$ is an effective divisor with $\operatorname{deg}\left(D_{2}^{\prime}\right)<\operatorname{deg}\left(D_{2}\right)$ and $\mathfrak{p}$ is a closed point on $Y$. By repeating the same argument that we used above, we get

$$
\chi\left(G, f^{*}\left(\mathscr{O}_{Y}(D)\right)\right)=\chi\left(G, f^{*}\left(\mathscr{O}_{Y}\left(D_{1}-D_{2}^{\prime}\right)\right)\right)-\operatorname{deg}(\mathfrak{p})[A[G]] .
$$

Definition 5.7. Assume now that $A=\mathbf{k}$ is a perfect field of characteristic $p$, and let $W$ denote the ring of Witt vectors of $\mathbf{k}$ of infinite length. Moreover, let $K$ denote the field of fractions of $W$. Let $M$ be a finitely generated cohomologically trivial $\mathbf{k}[G]$-module. Then $M$ is a projective $\mathbf{k}[G]$-module by [Chinburg 1994, Proposition 4.1(a)]. Hence $M$ is isomorphic to $P / p P$ for some finitely generated projective $W$-module $P$. The character of the $\bar{K}[G]$-module $\bar{K} \otimes_{W} P$ can be written in the form $\sum m_{\alpha} \alpha$, where the sum is over the set $R(G)$ of irreducible $\bar{K}$-valued characters of $G$. The integer $m_{\alpha}$ is independent of the choice of $P$. Let $\Delta(M)$ : $R(G) \rightarrow \mathbb{Z}$ be the function defined by the formula $\Delta(M)(\alpha)=m_{\alpha}$. This map extends uniquely to a homomorphism from $C T(A[G])$ to the group of $\mathbb{Z}$-valued functions on $R(G)$ which will be denoted by $\Delta$ as well. 
Suppose that $A$ is a finite extension of the field $B$. Then the restriction of operators from $A[G]$ to $B[G]$ induces a homomorphism $\operatorname{Res}_{A \rightarrow B}: C T(A[G]) \rightarrow$ $C T(B[G]))$. Assume now that $A$ is the finite field $\mathbb{F}_{q}$. Then every $\alpha \in R(G)$ can be considered as a representation of the absolute Galois group of the function field of $X$. In particular the $\epsilon$-constant $\epsilon(\alpha) \in \overline{\mathbb{Q}}_{p}$ is defined.

Theorem 5.8. $\Delta\left(\operatorname{Res}_{\mathbb{E}_{q} \rightarrow \mathbb{F}_{p}}\left(\chi\left(G, \mathcal{O}_{X}\right)\right)\right)(\alpha)=-d v_{q}\left(\epsilon\left(\alpha^{-1}\right)\right)$ for all $\left.\alpha \in R(G)\right)$.

Proof. This is a special case of [Chinburg 1994, Theorem 5.2].

Notation 5.9. Suppose now that $Y=\mathscr{C}$, and let $\mathscr{E}$ and $\Delta_{E}$ be the same as in the introduction. Assume that the ramification divisor of the cover $f: X \rightarrow Y$ has support disjoint from the conductor of $E$. Let $g^{\prime}: \mathscr{E}^{\prime} \rightarrow X$ be the base change of the elliptic fibration $g: \mathscr{E} \rightarrow Y$ with respect to the map $f$. Note that the $X$-scheme $\mathscr{E}^{\prime}$ is a relatively minimal regular model of the base change of $E$ to the function field of $X$. Moreover, $\mathscr{E}^{\prime}$ is equipped with a unique action of $G$ fixing the zero section such that $g^{\prime}$ is equivariant with respect to this action and the one on $X$.

Theorem 5.10. The $\mathbb{F}_{q}[G]$-module $H^{2}\left(\mathscr{E}^{\prime}, \mathscr{O}_{\mathscr{E}}\right)$ is cohomologically trivial and $\Delta\left(\operatorname{Res}_{\mathbb{F}_{q} \rightarrow \mathbb{F}_{p}}\left(\left[H^{2}\left(\mathscr{E}^{\prime}, \mathscr{O}_{\mathscr{E}^{\prime}}\right)\right]\right)\right)(\alpha)=\frac{1}{12} d \operatorname{deg}\left(\Delta_{E}\right)+d v_{q}\left(\epsilon\left(\alpha^{-1}\right)\right)$ for all $\alpha \in R(G)$. Proof. By Lemma 5.5, we have

$$
\chi\left(G, \mathscr{O}_{\mathscr{E}^{\prime}}\right)=\left[H^{0}\left(\mathscr{E}^{\prime}, \mathcal{O}_{\mathscr{E}^{\prime}}\right)\right]-\left[H^{1}\left(\mathscr{E}^{\prime}, \mathrm{O}_{\mathscr{E}^{\prime}}\right)\right]+\left[H^{2}\left(\mathscr{E}^{\prime}, \mathrm{O}_{\mathscr{C}^{\prime}}\right)\right] .
$$

By [Goldfeld and Szpiro 1995, Lemma 4], the map $\left(g^{\prime}\right)^{*}: \operatorname{Pic}^{0}(X) \rightarrow \operatorname{Pic}^{0}\left(\mathscr{E}^{\prime}\right)$ induced by Picard functoriality is an isomorphism. Because this map is equivariant with respect to the induced $G$-actions on $\operatorname{Pic}^{0}(X)$ and $\operatorname{Pic}^{0}\left(\mathscr{E}^{\prime}\right)$, we get that $H^{1}\left(\mathscr{E}^{\prime}, \mathscr{O}_{\mathscr{E}}\right)=H^{1}\left(X, \mathscr{O}_{X}\right)$ as $\mathbb{F}_{q}[G]$-modules, since these modules are isomorphic to the tangent spaces at the zero of the abelian varieties $\operatorname{Pic}^{0}\left(\mathscr{E}^{\prime}\right)$ and $\operatorname{Pic}^{0}(X)$, respectively. Obviously $H^{0}\left(\mathscr{E}^{\prime}, \mathcal{O}_{\mathscr{E}^{\prime}}\right)=H^{0}\left(X, \mathscr{O}_{X}\right)$ as $\mathbb{F}_{q}[G]$-modules, and hence from (13) and Lemma 5.5 we get

$$
\left[H^{2}\left(\mathscr{E}^{\prime}, \mathscr{O}_{\mathscr{E}}\right)\right]=\chi\left(G, \mathscr{O}_{\mathscr{E}}\right)-\chi\left(G, \mathscr{O}_{X}\right) .
$$

Let $\Omega_{\mathscr{E} / Y}^{1}$ and $\Omega_{\mathscr{E}^{\prime} / X}^{1}$ denote the sheaf of relative Kähler differentials of the $Y$ scheme $\mathscr{E}$ and that of the $X$-scheme $\mathscr{E}^{\prime}$. Let $\omega_{\mathscr{E} / Y}$ and $\omega_{\mathscr{E}} / X$ denote the pull-backs of $\Omega_{\mathscr{E} / Y}^{1}$ and $\Omega_{\mathscr{E}^{\prime} / X}^{1}$ with respect to the zero section. These sheaves are line bundles on $Y$ and $X$, respectively. Moreover, by Grothendieck's duality we have $\mathbb{R}^{1} g_{*}^{\prime}\left(\mathscr{O}_{\mathscr{E}}\right)=$ $\omega_{\mathscr{E}^{\prime} / X}^{\otimes-1}$. In particular, $\chi\left(G, R^{1} g_{*}^{\prime}\left(O_{\mathscr{E}^{\prime}}\right)\right)=-\chi\left(G, \omega_{\mathscr{E}^{\prime} / X}\right)$. Therefore, because all boundary maps in the spectral sequence $H^{p}\left(Y, R^{q} g_{*}^{\prime}\left(\mathcal{O}_{\mathscr{E}^{\prime}}\right)\right) \Rightarrow H^{p+q}\left(\mathscr{E}^{\prime}, \mathcal{O}_{\mathscr{E}}\right)$ are $\mathbb{F}_{q}[G]$-linear, we get from Lemma 5.5 that

$$
\chi\left(G, \mathscr{O}_{\mathscr{E}^{\prime}}\right)=\chi\left(G, \mathscr{O}_{X}\right)-\chi\left(G, R^{1} g_{*}^{\prime}\left(\mathcal{O}_{\mathscr{E}^{\prime}}\right)\right)=\chi\left(G, \mathscr{O}_{X}\right)-\chi\left(G, \omega_{\mathscr{C}^{\prime} / X}^{\otimes-1}\right) .
$$


Combining (14) and (15), we get that

$$
\left[H^{2}\left(\mathscr{E}^{\prime}, \mathcal{O}_{\mathscr{C}^{\prime}}\right)\right]=-\chi\left(G, \omega_{\mathscr{C}^{\prime} / X}^{\otimes-1}\right) .
$$

By definition, $\Delta_{E}$ is the zero divisor of a nonzero section of $\omega_{\mathscr{E} / Y}^{\otimes 12}$. Therefore $\operatorname{deg}\left(\Delta_{E}\right)=12 \operatorname{deg}\left(\omega_{\mathscr{E} / Y}\right)$. Moreover $\omega_{\mathscr{E}^{\prime} / X}=f^{*}\left(\omega_{\mathscr{E} / Y}\right)$. Hence we see from (16) and Lemma 5.6 that

$$
\left[H^{2}\left(\mathscr{E}, \mathscr{O}_{\mathscr{E}}\right)\right]=\frac{1}{12} \operatorname{deg}\left(\Delta_{E}\right)\left[\mathbb{F}_{q}[G]\right]-\chi\left(G, \mathscr{O}_{X}\right) .
$$

The claim now follows from Theorem 5.8.

\section{Slope estimates}

Definition 6.1. Let $\sigma: W \rightarrow W$ denote the absolute Frobenius automorphism. Let $W((V))$ denote the $W$-algebra of formal Laurent series $\sum_{i \geq n} a_{i} V^{i}$, where $a_{i} \in W$ and $n \in \mathbb{Z}$ are arbitrary, with the usual addition and with multiplication defined by

$$
\left(\sum_{i \geq n} a_{i} V^{i}\right) \cdot\left(\sum_{j \geq m} b_{j} V^{j}\right)=\sum_{k \geq n+m}\left(\sum_{i+j=k} a_{i} \sigma^{-i}\left(b_{j}\right)\right) V^{k} .
$$

Moreover, let $W[V]$ and $W \llbracket V \rrbracket$ denote the subring of $W((V))$ consisting of polynomials and formal power series in the variable $V$, respectively. Let $M$ be a module over the ring $W[V]$. Then the kernel and the cokernel of the multiplication by $V: M \rightarrow M$ are $W$-modules, and we define

$$
\chi(M)=\text { length }_{W}(\operatorname{Ker}(V))-\text { length }_{W}(\operatorname{Coker}(V)),
$$

provided both numbers on the right are finite. Let $W[V, F]$ denote the ring generated by the variable $V$ over $W$ subject to the relations $V F=F V=p, F c=\sigma(c) F$ and $V c=\sigma^{-1}(c) V$, for every $c \in W$. For every module $M$ over the Dieudonné ring $W[F, V]$ that is free and finitely generated as a $W$-module, the tensor product $M \otimes_{W} K$ is an $F$-isocrystal over $W$ with respect to multiplication by $F \otimes_{W} \mathrm{id}_{K}$. For every $F$-isocrystal $M$ over $W$, let $l(M)$ denote $\sum m_{i}\left(1-\lambda_{i}\right)$, where the $\lambda_{i}$ are the slopes of $M$ and $m_{i}$ is the multiplicity of $\lambda_{i}$.

Lemma 6.2. (i) If $0 \rightarrow M^{\prime} \rightarrow M \rightarrow M \rightarrow 0$ is a short exact sequence of $W[V]-$ modules, then $\chi(M)$ is defined if $\chi\left(M^{\prime}\right)$ and $\chi\left(M^{\prime \prime}\right)$ are defined and

$$
\chi(M)=\chi\left(M^{\prime}\right)+\chi\left(M^{\prime \prime}\right) .
$$

(ii) If $M$ is a module over the ring $W[F, V]$ that is free and finitely generated as $a W$-module, then $\chi(M)=-l\left(M \otimes_{W} K\right)$.

(iii) If $M$ is a finitely generated torsion $W \llbracket V \rrbracket-m o d u l e$, then

$$
\chi(M)=- \text { length }_{W((V))} M \otimes_{W \llbracket V \rrbracket} W((V)) .
$$


Proof. This is [Milne 1975, Lemma 7.2].

Definition 6.3. Let $G$ be a finite abelian group whose order is not divisible by $p$, and assume that $\mathbf{k}$ is algebraically closed. Then every $\alpha \in R(G)$ takes values in $W$, so the element $\pi_{\alpha}=\sum_{g \in G} \alpha(g)^{-1} g \in W[G]$ is well-defined. For every $W[G]$-module $M$, let $M^{\alpha}$ denote the sub- $W[G]$-module $\pi_{\alpha} M$. Finally, for every $F$-isocrystal $M$ over $W$ and real numbers $a \leq b$, let $M_{[a, b)}$ denote the maximal subquotient of $M$ with slopes in the interval $[a, b)$.

Let $X$ be a smooth projective variety defined over $\mathbf{k}$, and assume that $G$ acts on $X$. Let $H^{m}(X / W)$ denote the $m$-th crystalline cohomology group of $W$. Then $W[G]$ acts on $H^{m}(X / W)$ and $H^{m}\left(X, \mathcal{O}_{X}\right)$ by functoriality for every $m$.

Proposition 6.4. $l\left(H^{r}(X / W)^{\alpha} \otimes_{W} K_{[0,1)}\right) \leq \operatorname{dim}_{\mathbf{k}}\left(H^{r}\left(X, O_{X}\right)^{\alpha}\right)$, for every $r \in \mathbb{N}$ and $\alpha \in R(G)$.

Proof. Let $H^{r}(X, W)$ denote the $r$-th Witt vector cohomology group of the variety $X$. This is a $W[V]$-module and there is a long exact sequence

$$
\cdots \rightarrow H^{r-1}\left(X, \mathrm{O}_{X}\right) \rightarrow H^{r}(X, W) \stackrel{V}{\rightarrow} H^{r}(X, W) \rightarrow H^{r}\left(X, \mathrm{O}_{X}\right) \rightarrow \cdots
$$

The group $G$ acts on all cohomology groups in this sequence, and the maps are equivariant with respect to this action, so there is a long exact sequence

$$
\cdots \rightarrow H^{r-1}\left(X, \mathrm{O}_{X}\right)^{\alpha} \rightarrow H^{r}(X, W)^{\alpha} \stackrel{V}{\rightarrow} H^{r}(X, W)^{\alpha} \rightarrow H^{r}\left(X, \mathrm{O}_{X}\right)^{\alpha} \rightarrow \cdots
$$

Because $X$ is projective, the vector spaces $H^{r}\left(X, O_{X}\right)^{\alpha}$ have finite dimension. Therefore $\chi\left(H^{r}(X, W)^{\alpha}\right)$ is well-defined and

$$
\chi\left(H^{r}(X, W)^{\alpha}\right) \geq-\operatorname{dim}_{\mathbf{k}}\left(H^{r}\left(X, O_{X}\right)^{\alpha}\right) .
$$

Write

$$
H^{r}(X, W)_{t}^{\alpha}=\operatorname{Ker}\left(H^{r}(X, W)^{\alpha} \rightarrow H^{r}(X, W)^{\alpha} \otimes_{W} K\right) .
$$

Then $H^{r}(X, W)_{t}^{\alpha}$ is a torsion $W \llbracket V \rrbracket$-module, and there is a short exact sequence of $W[V]$-modules

$$
0 \rightarrow H^{r}(X, W)_{t}^{\alpha} \rightarrow H^{r}(X, W)^{\alpha} \rightarrow H^{r}(X, W)_{c t}^{\alpha} \rightarrow 0
$$

such that $H^{r}(X, W)_{c t}^{\alpha}$ is a module over the Dieudonné ring $W[F, V]$ that is free and finitely generated as a $W$-module. Therefore by of Lemma 6.2(i), we have

$$
\chi\left(H^{r}(X, W)^{\alpha}\right)=\chi\left(H^{r}(X, W)_{t}^{\alpha}\right)+\chi\left(H^{r}(X, W)_{c t}^{\alpha}\right) .
$$

Because the slope spectral sequence degenerates modulo torsion [Illusie 1979, théorème 3.2], there is an isomorphism between the $F$-isocrystals $H^{r}(X, W) \otimes_{W} K$ and $H^{r}(X / W) \otimes K_{[0,1)}$. Since this isomorphism is equivariant with respect to the 
action of $G$, the $F$-isocrystals $H^{r}(X, W)^{\alpha} \otimes_{W} K$ and $H^{r}(X / W)^{\alpha} \otimes_{W} K_{[0,1)}$ are also isomorphic. Hence

$$
\chi\left(H^{r}(X, W)_{c t}^{\alpha}\right)=-l\left(H^{r}(X, W)_{c t}^{\alpha} \otimes_{W} K\right)=-l\left(H^{r}(X / W)^{\alpha} \otimes_{W} K_{[0,1)}\right)
$$

by Lemma 6.2(ii). According to (iii), the number $\chi\left(H^{r}(X, W)_{t}^{\alpha}\right)$ is not positive. Hence (17), (18), and (19) imply that

$$
-l\left(H^{r}(X / W)^{\alpha} \otimes_{W} K_{[0,1)}\right) \geq-\operatorname{dim}_{\mathbf{k}}\left(H^{r}\left(X, \mathscr{O}_{X}\right)^{\alpha}\right) .
$$

Notation 6.5. Assume now that $\mathbf{k}$ is the algebraic closure of $\mathbb{F}_{q}$, and for every $\operatorname{Spec}\left(\mathbb{F}_{q}\right)$-scheme $S$, let $\bar{S}$ denote its base change to $\operatorname{Spec}(\mathbf{k})$. Moreover let $F$ : $\bar{S} \rightarrow \bar{S}$ denote the Frobenius relative to $\mathbb{F}_{q}$ for every such $S$. Assume now that $X$ is a smooth projective variety defined over k equipped with an action of $G$. Recall that we've chosen a prime $l \neq p$. Now fix an isomorphism $v: \overline{\mathbb{Q}}_{l} \rightarrow \overline{\mathbb{Q}}_{p}$. Since the group $G$ acts on the étale cohomology group $H^{m}\left(\bar{X}, \overline{\mathbb{Q}}_{l}\right)$, we may consider the latter as a $\mathbb{W}[G]$-module if we identify $\overline{\mathbb{Q}}_{l}$ and $\overline{\mathbb{Q}}_{p}$ via $v$. Then the map $F^{*}$ : $H^{m}\left(\bar{X}, \overline{\mathbb{Q}}_{l}\right) \rightarrow H^{m}\left(\bar{X}, \overline{\mathbb{Q}}_{l}\right)$ induced by the Frobenius morphism $F$ commutes with the action of $G$, so $H^{m}\left(\bar{X}, \overline{\mathbb{Q}}_{l}\right)^{\alpha}$ is an $F^{*}$-invariant subspace.

Lemma 6.6. $l_{q}\left(\operatorname{det}\left(1-F^{*} t \mid H^{m}\left(\bar{X}, \overline{\mathbb{Q}}_{l}\right)^{\alpha}\right)\right)=l\left(H^{m}(\bar{X} / W)^{\alpha} \otimes_{W} K_{[0,1)}\right)$.

Proof. For every $g \in G$, let the same symbol denote the base change $\bar{X} \rightarrow \bar{X}$ to $\operatorname{Spec}(\mathbf{k})$ of the automorphism $X \rightarrow X$ furnished by the given action of $G$ on $X$. For every positive integer $n$, the composition

$$
g \circ F^{[n]}=g \circ \underbrace{F \circ \cdots \circ F}_{n \text { times }}
$$

induces the zero map on tangent spaces, and hence the Lefschetz trace formula applied to $g \circ F^{[n]}$ both in the $l$-adic and the crystalline cohomology theories implies

$$
\begin{aligned}
\sum_{m=0}^{2 \operatorname{dim}(\bar{X})}(-1)^{m} \operatorname{Tr}\left(1-g^{*}\right. & \left.\left(F^{*}\right)^{n} \mid H^{m}\left(\bar{X}, \overline{\mathbb{Q}}_{l}\right)\right) \\
& =\sum_{m=0}^{2 \operatorname{dim}(\bar{X})}(-1)^{m} \operatorname{Tr}\left(1-g^{*}\left(F^{*}\right)^{n} \mid H^{m}(\bar{X} / W) \otimes_{W} K\right) .
\end{aligned}
$$

Let $l_{m}(\alpha, t)$ and $c_{m}(\alpha, t)$ denote the polynomials

$$
\operatorname{det}\left(1-F^{*} t \mid H^{m}\left(\bar{X}, \overline{\mathbb{Q}}_{l}\right)^{\alpha}\right) \quad \text { and } \quad \operatorname{det}\left(1-F^{*} t \mid H^{m}(\bar{X} / W) \otimes_{W} K^{\alpha}\right) .
$$

By the orthogonality of characters, (20) implies that

$$
\prod_{m=0}^{2 \operatorname{dim}(\bar{X})} l_{m}(\alpha, t)^{(-1)^{m}}=\prod_{m=0}^{2 \operatorname{dim}(\bar{X})} c_{m}(\alpha, t)^{(-1)^{m}} .
$$


By Deligne's purity theorem and [Katz and Messing 1974, Theorem 1], the reciprocal roots of the polynomials $l_{m}(\alpha, t)$ and $c_{m}(\alpha, t)$ are Weil numbers with squared complex norm $q^{m}$. Hence there are no cancellations in the alternating products in (21). Therefore $l_{m}(\alpha, t)=c_{m}(\alpha, t)$ for every $m$. Let $\lambda_{1}, \lambda_{2}, \ldots, \lambda_{k}$ be the eigenvalues of $F^{*}$ considered as a linear transformation of $H^{m}(\bar{X} / W) \otimes_{W} K^{\alpha}$. The claim now follows from the fact that $v_{q}\left(\lambda_{1}\right), v_{q}\left(\lambda_{2}\right), \ldots, v_{q}\left(\lambda_{k}\right)$ are the slopes of the $F$-isocrystal $H^{m}(\bar{X} / W) \otimes_{W} K^{\alpha}$.

Notation 6.7. Let us consider now the same situation as in the introduction. Fix a tamely ramified character $\alpha \in \mathbb{X}(\mathfrak{n})$ and let $\pi: X \rightarrow \mathscr{C}$ be the Galois cover corresponding to the extension $F^{\prime} \mid F$, where $F^{\prime}$ is the subfield of $\bar{F}$ fixed by the kernel of $\alpha$. Let $G$ denote the Galois group of the cover $\pi$ and let $g^{\prime}: \mathscr{E}^{\prime} \rightarrow X$ denote the base change of $g: \mathscr{E} \rightarrow \mathscr{C}$ with respect to the map $f$ as in Notation 5.9. We will also keep on using the notation introduced in Notation 6.5.

Lemma 6.8. $l_{q}\left(L\left(\sigma_{E} \otimes \alpha, t\right)\right)=l\left(H^{2}\left(\overline{\mathscr{E}^{\prime}} / W\right)^{\alpha} \otimes_{W} K_{[0,1)}\right)$.

Proof. For every morphism $m: R \rightarrow S$ of $\operatorname{Spec}\left(\mathbb{F}_{q}\right)$-schemes, let $\bar{m}: \bar{R} \rightarrow \bar{S}$ denote the base change to $\operatorname{Spec}(\mathbf{k})$. The Leray spectral sequence $H^{p}\left(\bar{X}, R^{q} \bar{g}_{*}^{\prime}\left(\overline{\mathbb{Q}}_{l}(1)\right)\right) \Rightarrow$ $H^{p+q}\left(\overline{\mathscr{E}}^{\prime}, \overline{\mathbb{Q}}_{l}(1)\right)$ furnishes an injection $\zeta: H^{1}\left(\bar{X}, R^{1} \bar{g}_{*}^{\prime}\left(\overline{\mathbb{Q}}_{l}(1)\right)\right) \rightarrow H^{2}\left(\overline{\mathscr{C}}^{\prime}, \overline{\mathbb{Q}}_{l}(1)\right)$, and the image of this map is the orthogonal complement of the $\overline{\mathbb{Q}}_{l}$-linear subspace $V$ spanned by the Chern classes of the zero section and the fibres of the elliptic fibration $\overline{\mathscr{E} \prime} \rightarrow \overline{\mathscr{C}}$, considered here as divisors on the surface $\overline{\mathscr{E}}^{\prime}$, with respect to the cup product pairing. The cohomology group $H^{1}\left(\bar{X}, R^{1} \bar{g}_{*}^{\prime}\left(\overline{\mathbb{Q}}_{l}(1)\right)\right)$ is naturally equipped with a $G$-action because the map $\bar{g}^{\prime}$ is equivariant with respect to the action of $G$ on $\overline{\mathscr{E} \prime}$ and $\bar{X}$, respectively. Moreover $\zeta$ is $G$-linear. Hence we have an isomorphism

$$
H^{1}\left(\bar{X}, R^{1} \bar{g}_{*}^{\prime}\left(\overline{\mathbb{Q}}_{l}\right)\right)^{\alpha} \oplus V^{\alpha}(-1) \cong H^{2}\left(\overline{\mathscr{C}}^{\prime}, \overline{\mathbb{Q}}_{l}\right)^{\alpha} .
$$

Note that the cohomology group $H^{1}\left(\bar{X}, R^{1} \bar{g}_{*}^{\prime}\left(\overline{\mathbb{Q}}_{l}\right)\right)$ is equipped with the action of a Frobenius operator $F^{*}$ that commutes with the action of $G$, and that the isomorphism in (22) respects the action of the operator $F^{*}$ on both sides. Moreover, for every eigenvalue $\lambda$ of $F^{*}$ on $V(-1)$, we have $v_{q}(\lambda)=1$, and hence

$$
l_{q}\left(\operatorname{det}\left(1-F^{*} t \mid H^{1}\left(\bar{X}, R^{1} \bar{g}_{*}^{\prime}\left(\overline{\mathbb{Q}}_{l}\right)\right)^{\alpha}\right)\right)=l_{q}\left(\operatorname{det}\left(1-F^{*} t \mid H^{2}\left(\overline{\mathscr{E}}^{\prime}, \overline{\mathbb{Q}}_{l}\right)^{\alpha}\right)\right) .
$$

We may consider $\alpha$ a lisse $l$-adic sheaf on $\operatorname{Spec}(F)$. By slight abuse of notation, let $\alpha$ also denote the pull-back onto $\overline{\mathscr{C}}$ of the direct image of this sheaf $\alpha$ with respect to the open immersion $\operatorname{Spec}(F) \rightarrow \mathscr{C}$. Because the Galois representation $H^{1}\left(E_{\bar{F}}, \overline{\mathbb{Q}}_{l}\right)$ is absolutely irreducible and self-dual, we have

$$
H^{0}\left(\overline{\mathscr{C}}, R^{1} \bar{g}_{*}\left(\overline{\mathbb{Q}}_{l}\right) \otimes \alpha\right)=H^{2}\left(\overline{\mathscr{C}}, R^{1} \bar{g}_{*}\left(\overline{\mathbb{Q}}_{l}\right) \otimes \alpha\right)=0 .
$$


Moreover, $H^{1}\left(\overline{\mathscr{C}}, R^{1} \bar{g}_{*}\left(\overline{\mathbb{Q}}_{l}\right) \otimes \alpha\right)=H^{1}\left(\bar{X}, R^{1} \bar{g}_{*}^{\prime}\left(\overline{\mathbb{Q}}_{l}\right)\right)^{\alpha}$ by the degeneration of the Hochschild-Serre spectral sequence. Hence by the Grothendieck-Verdier trace formula,

$$
\begin{aligned}
l_{q}\left(L\left(\sigma_{E} \otimes \alpha, t\right)\right)=l_{q}\left(\operatorname{det}\left(1-F^{*} t \mid H^{1}\left(\bar{X}, R^{1} \bar{g}_{*}^{\prime}\left(\overline{\mathbb{Q}}_{l}\right)\right)^{\alpha}\right)\right. \\
\quad=l_{q}\left(\operatorname{det}\left(1-F^{*} t \mid H^{2}\left(\overline{\mathscr{E}}^{\prime}, \overline{\mathbb{Q}}_{l}\right)^{\alpha}\right)\right) .
\end{aligned}
$$

The claim now follows from Lemma 6.6.

Theorem 6.9. Assume that $p$ does not divide the order of $\operatorname{Pic}_{0}(\mathscr{C})\left(\mathbb{F}_{q}\right)$. Then

$$
m(E) \leq d\left(\frac{1}{12} \operatorname{deg}\left(\Delta_{E}\right)+g-1\right) .
$$

Proof. Let $\alpha \in \mathbb{X}(\mathfrak{n})$ be arbitrary, and let $C(\alpha)$ denote the set of all characters $\beta: \operatorname{Gal}\left(F^{\prime} \mid F\right) \rightarrow \overline{\mathbb{Q}}_{p}^{*}$ that are conjugate to $\alpha$ under the action of the absolute Galois group $\operatorname{Gal}\left(\overline{\mathbb{Q}}_{p} \mid \mathbb{Q}_{p}\right)$ on $\overline{\mathbb{Q}}_{p}$. For every $\beta \in C(\alpha)$, we have

$$
l_{q}\left(L\left(\sigma_{E} \otimes \beta, t\right)\right) \leq \operatorname{dim}_{\mathbf{k}}\left(H^{2}\left(\overline{\mathscr{C}}^{\prime}, \mathcal{O}_{\overline{\bar{C}^{\prime}}}\right)^{\beta}\right)=\operatorname{dim}_{\mathbf{k}}\left(H^{2}\left(\mathscr{E}^{\prime}, \mathcal{O}_{\mathscr{E}^{\prime}}\right) \otimes_{\mathbb{F}_{q}} \mathbf{k}^{\beta}\right)
$$

by Proposition 6.4 and Lemma 6.8. By [Chinburg 1994, Remark 4.5],

$$
\Delta\left(\operatorname{Res}_{\mathbb{F}_{q} \rightarrow \mathbb{F}_{p}}\left(\left[H^{2}\left(\mathscr{E}^{\prime}, \mathscr{O}_{\mathscr{E}^{\prime}}\right)\right]\right)(\alpha)=\frac{d}{|C(\alpha)|} \sum_{\beta \in C(\alpha)} \operatorname{dim}_{\mathbf{k}}\left(H^{2}\left(\mathscr{E}^{\prime}, \mathcal{O}_{\mathscr{E}^{\prime}}\right) \otimes_{\mathbb{F}_{q}} \mathbf{k}^{\beta}\right)\right.
$$

Because the action of $\operatorname{Gal}\left(\overline{\mathbb{Q}}_{p} \mid \mathbb{Q}_{p}\right)$ on $\overline{\mathbb{Q}}_{p}$ leaves the valuation $v_{q}$ invariant, we have

$$
l_{q}\left(L\left(\sigma_{E} \otimes \beta, t\right)\right)=l_{q}\left(L\left(\sigma_{E} \otimes \alpha, t\right)\right)
$$

for every $\beta \in C(\alpha)$. Hence (23), (24) and Theorem 5.10 imply that

$$
\begin{aligned}
l_{q}\left(L\left(\sigma_{E} \otimes \alpha, t\right)\right) & =\frac{1}{|C(\alpha)|} \sum_{\beta \in C(\alpha)} l_{q}\left(L\left(\sigma_{E} \otimes \beta, t\right)\right) \\
& \leq \frac{1}{|C(\alpha)|} \sum_{\beta \in C(\alpha)} \operatorname{dim}_{\mathbf{k}}\left(H^{2}\left(\mathscr{E}^{\prime}, \mathscr{O}_{\mathscr{C}^{\prime}}\right) \otimes_{\mathbb{F}_{q}} \mathbf{k}^{\beta}\right) \\
& =\frac{1}{d} \cdot \Delta\left(\operatorname{Res}_{\mathbb{F}_{q} \rightarrow \mathbb{F}_{p}}\left(\left[H^{2}\left(\mathscr{C}^{\prime}, \mathscr{O}_{\mathscr{C}^{\prime}}\right)\right]\right)(\alpha)\right. \\
& =\frac{1}{12} \operatorname{deg}\left(\Delta_{E}\right)+v_{q}\left(\epsilon\left(\alpha^{-1}\right)\right) .
\end{aligned}
$$

The claim now follows from Proposition 4.5.

Definition 6.10. Following [Mazur 1972], we will call a smooth projective variety $V$ defined over a perfect field of characteristic $p$ ordinary in dimension $n$ if the $n$-dimensional Newton and Hodge polygons of $V$ agree.

Theorem 6.11. Assume that the elliptic surface $\mathscr{E}$ is ordinary in dimension 2 . Then

$$
m(E) \geq d\left(\frac{1}{12} \operatorname{deg}\left(\Delta_{E}\right)+g-1\right) .
$$


Proof. By Deligne's purity theorem, we have $\epsilon(1)= \pm q^{g-1}$, where 1 denotes the trivial representation. Hence in the special case when $G$ is the trivial group, that is, the cover $f$ is the identity map of $\mathscr{C}$ onto itself, Theorem 5.10 says that

$$
\operatorname{dim}_{\mathbb{F}_{q}} H^{2}\left(\mathscr{E}, \mathscr{O}_{\mathscr{E}}\right)=\frac{1}{12} \operatorname{deg}\left(\Delta_{E}\right)+g-1 .
$$

Because $\mathscr{E}$ is ordinary in dimension 2, we have

$$
l_{q}(L(E, t))=l_{q}\left(\operatorname{det}\left(1-F^{*} t \mid H^{2}\left(\overline{\mathscr{E}}, \mathbb{Q}_{l}\right)\right)\right)=\operatorname{dim}_{\mathbb{F}_{q}} H^{2}\left(\mathscr{E}, \mathscr{O}_{\mathscr{E}}\right)
$$

by definition. The claim now follows from Proposition 4.5.

Remark 6.12. It is known that a smooth projective variety $V$ is ordinary if $V$ is a generic curve of genus $g$ [Faber and van der Geer 2004; Miller 1972], a generic abelian variety of dimension $d$ equipped with a polarisation of degree $r$ [Mumford 1969; Norman and Oort 1980], or a generic complete intersection of multidegree $\left(a_{1}, a_{2}, \ldots, a_{n}\right)$ [Illusie 1990]. It is natural to expect that the same holds for elliptic surfaces with a section. More precisely, one might conjecture the following. Assume that $p>3$, let $N \geq 2$ be a positive integer, let $g \in \mathbb{N}$ and let $M_{g, N, p}$ denote the coarse moduli representing the functor that associates to every scheme $T$ over $\operatorname{Spec}\left(\mathbb{F}_{p}\right)$ the set of isomorphism classes of smooth families of elliptic surfaces over a smooth curve of genus $g$ over $T$ with discriminant of degree $12 \mathrm{~N}$ in all geometric fibres over $T$, constructed in [Seiler 1987]. Then I expect that for every $p, g$ and $N$ there is a nonempty open subscheme $U$ of $\mathcal{M}_{g, N, p}$ such that for every geometric point of $U$, the corresponding elliptic surface is ordinary. Of course it is enough to show that there is a geometric point of $M_{g, N, p}$ such that the corresponding elliptic surface is ordinary in dimension two.

\section{An upper bound in terms of the conductor}

Notation 7.1. For every elliptic curve $E$ defined over a field $K$ of characteristic $p$, let $E^{(p)}$ denote pull-back of $E$ with respect to the Frobenius map $K \rightarrow K$ (given by $x \mapsto x^{p}$ ). We will call $E^{(p)}$ the Frobenius twist of $E$. The elliptic curve $E^{(p)}$ is in fact defined over the subfield $K^{p}$ of $p$-th powers. The absolute Frobenius $\mathbf{F}: E \rightarrow E^{(p)}$ is an isogeny defined over the field $K$. Finally, for every cohomology class $c \in H^{1}(K, \operatorname{Aut}(E))$, let $E_{c}$ denote twist of $E$ by $c$.

Definition 7.2. Let $K$ be as above and let $E$ be an elliptic curve defined over $K$ such that $j(E) \neq 0,1728$. Because $j\left(E^{(p)}\right)=j(E)^{p}$, we have $j\left(E^{(p)}\right) \neq 0,1728$. Hence by [Silverman 1986, Proposition 1.2(c)], the groups $\operatorname{Aut}(E)$ and $\operatorname{Aut}\left(E^{(p)}\right)$ are both equal to multiplication by \pm 1 . Therefore there is a unique isomorphism $f: \operatorname{Aut}(E) \rightarrow \operatorname{Aut}\left(E^{(p)}\right)$ such that $\mathbf{F} \circ \phi=f(\phi) \circ \mathbf{F}$ for every $\phi \in \operatorname{Aut}(E)$. Let $f_{*}: H^{1}(K, \operatorname{Aut}(E)) \rightarrow H^{1}\left(K, \operatorname{Aut}\left(E^{(p)}\right)\right)$ denote the isomorphism induced by the identification $f$. 
The next two results will be useful, and they seem not to be recorded in the literature.

Lemma 7.3. Assume that $j(E) \neq 0,1728$. Then the elliptic curves $\left(E_{c}\right)^{(p)}$ and $\left(E^{(p)}\right)_{f_{*}(c)}$ are isomorphic over $K$ for every $c \in H^{1}(K, \operatorname{Aut}(E))$.

Proof. For every scheme $X$ over $\operatorname{Spec}(K)$, let $\bar{X}$ denote $X \times_{\operatorname{Spec}(K)} \operatorname{Spec}(\bar{K})$. For every $\gamma \in \operatorname{Gal}(\bar{K} \mid K)$ and $X$ as above, let the symbol $\gamma$ also denote the unique endomorphism of $\bar{X}$ that makes the following diagram commutative:

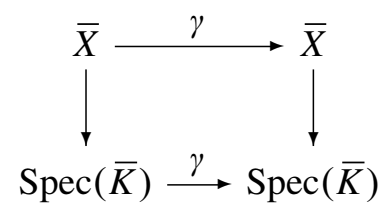

Fix an isomorphism $\phi: \bar{E} \rightarrow \overline{E_{c}}$ over $\bar{K}$. Then for every $\gamma \in \operatorname{Gal}(\bar{K} \mid K)$, the pull-back of the diagram

$$
\bar{E} \stackrel{\phi}{\longrightarrow} \overline{E_{c}} \stackrel{\gamma}{\longrightarrow} \overline{E_{c}} \stackrel{\phi^{-1}}{\longrightarrow} \bar{E} \stackrel{\gamma^{-1}}{\longrightarrow} \bar{E}
$$

with respect to the Frobenius map $x \mapsto x^{p}$ of $\operatorname{Spec}(\bar{K})$ is

$$
\overline{E^{(p)}} \stackrel{\psi}{\longrightarrow} \overline{\left(E_{c}\right)^{(p)}} \stackrel{\gamma}{\longrightarrow} \overline{\left(E_{c}\right)^{(p)}} \stackrel{\psi^{-1}}{\longrightarrow} \overline{E^{(p)}} \stackrel{\gamma^{-1}}{\longrightarrow} \overline{E^{(p)}},
$$

where $\psi: \overline{E^{(p)}} \rightarrow \overline{\left(E_{c}\right)^{(p)}}$ is the unique isomorphism such that $\mathbf{F} \circ \phi=\psi \circ \mathbf{F}$. The Aut $(E)$-valued function $\gamma \mapsto \gamma^{-1} \circ \phi^{-1} \circ \gamma \circ \phi$ on $\operatorname{Gal}(\bar{F} \mid F)$ is a cocycle that represents $c$. Note that

$$
\mathbf{F} \circ \gamma^{-1} \circ \phi^{-1} \circ \gamma \circ \phi=\gamma^{-1} \circ \psi^{-1} \circ \gamma \circ \psi \circ \mathbf{F}
$$

for all $\gamma \in \operatorname{Gal}(\bar{F} \mid F)$ ), and therefore the function $\gamma \mapsto \gamma^{-1} \circ \psi^{-1} \circ \gamma \circ \psi$ is a cocycle that represents $f_{*}(c)$. The claim is now clear.

Proposition 7.4. Let $K$ be a field of characteristic $p$, and let $E$ be an elliptic curve defined over $K$. Assume that $j(E) \neq 0,1728$ and $j(E) \in K^{p}$. Then $E$ is isomorphic to the Frobenius twist of an elliptic curve $E^{\prime}$ defined over $K$.

Proof. Let $\lambda \in K$ be the unique $p$-th root of $j(E)$. By [Silverman 1986, Proposition 1.1], there is an elliptic curve $\widetilde{E}$ defined over $K$ such that $j(\widetilde{E})=\lambda$. Then the Frobenius twist $\widetilde{E}^{(p)}$ of $\widetilde{E}$ is defined over $K$ and has the same $j$-invariant as $E$. Hence by the theory of twists, there is a cohomology class $c \in H^{1}\left(K, \operatorname{Aut}\left(\widetilde{E}^{(p)}\right)\right)$ such that $E$ is the twist of $\widetilde{E}^{(p)}$ by $c$. Let $E^{\prime}$ be the twist of $\widetilde{E}$ by $f_{*}^{-1}(c)$. By Lemma 7.3, $E$ is isomorphic to the Frobenius twist of $E^{\prime}$.

Now let $K$ denote the function field of a smooth, projective, geometrically irreducible curve $X$ defined over a perfect field of characteristic $p$. 
Corollary 7.5. Let $E$ be a nonisotrivial elliptic curve defined over $K$. Then $E$ is isogenous to an elliptic curve $E^{\prime}$ defined over $K$ with the property $j\left(E^{\prime}\right) \notin K^{p}$.

Proof. By assumption, $j(E)$ does not lie in the constant field of $K$, and hence there are a $\lambda \in K$ and natural number $n$ such that $\lambda \notin K^{p}$ and $\lambda^{p^{n}}=j(E)$. By Proposition 7.4, there is an elliptic curve $E^{\prime}$ defined over $K$ such that $j\left(E^{\prime}\right)=\lambda$ and $E$ is the $n$-fold Frobenius twist of $E^{\prime}$. In particular, $E$ and $E^{\prime}$ are isogenous.

For every elliptic curve $E$ defined over $K$, let $\Delta_{E}$ denote the discriminant of a relatively minimal elliptic surface $\mathscr{E} \rightarrow X$ whose generic fibre is $E$. Then $\Delta_{E}$ is an effective divisor on the curve $X$. We say that a nonisotrivial elliptic curve $E$ is minimal in its isogeny class if $\operatorname{deg}\left(\Delta_{E}\right)=\min \left(\operatorname{deg}\left(\Delta_{E^{\prime}}\right)\right)$, where $E^{\prime}$ is any elliptic curve defined over $K$ isogenous to $E$. Let $\mathfrak{n}$ denote the conductor of $E$.

Theorem 7.6 (Pesenti-Szpiro). Assume that $E$ is a nonisotrivial elliptic curve that is minimal in its isogeny class. Then

$$
\operatorname{deg}\left(\Delta_{E}\right) \leq 6(\operatorname{deg}(\mathfrak{n})+2 g-2) .
$$

Proof. By Corollary 7.5, the claim follows at once from [Pesenti and Szpiro 2000, théorème 0.1$]$ and the isogeny-invariance of the conductor.

Let us return to the situation in the introduction. Because in each isogeny class of nonisotrivial elliptic curves there is an elliptic curve that is minimal, Theorem 7.6 combined with Theorem 1.3 has the following immediate corollary:

Corollary 7.7. Assume that $p$ does not divide the order of $\operatorname{Pic}_{0}(\mathscr{C})\left(\mathbb{F}_{q}\right)$. Then

$$
m(E) \leq d\left(\frac{1}{2} \operatorname{deg}(\mathfrak{n})+2 g-2\right) .
$$

Remark 7.8. Note that this inequality is significantly weaker than Theorem 1.3 because the Pesenti-Szpiro inequality fails to be an equality in general. For example, in the special case of the elliptic curve $E$ of Theorem 1.4, a fast inspection of [Ulmer 2002, 2.2 and 2.3] reveals that its conductor $\mathfrak{n}$ is the sum of the prime divisors of the polynomial $T\left(1-2^{4} 3^{3} T^{n}\right)$. Because by the assumptions of Theorem 1.4 the prime $p$ does not divide $2^{4} 3^{3} n$, the greatest common divisor of $1-2^{4} 3^{3} T^{n}$ and its derivative is

$$
\left(1-2^{4} 3^{3} T^{n},-n 2^{4} 3^{3} T^{n-1}\right)=(1),
$$

so we get that the polynomial $1-2^{4} 3^{3} T^{n}$ is square-fee and therefore $\operatorname{deg}(\mathfrak{n})=n+1$. Hence in this case Corollary 7.7 says that

$$
m(E) \leq \frac{1}{2} n-\frac{3}{2} .
$$

On the other hand, we will see in the next section that in this case Theorem 1.3 says that

$$
m(E) \leq \frac{1}{6} n-1
$$


and the two sides above are actually equal, by Theorem 1.4.

Remark 7.9. Corollary 7.7 was already proved in the special case when $\mathscr{C}=\mathbb{P}_{\mathbb{F}_{q}}^{1}$ and $\mathfrak{n}$ is square-free by Tan [1993]. His strategy is similar to ours in reducing the result to estimates of the $p$-adic valuations of coefficients of twisted $L$-functions. For the latter he uses the Grothendieck-Ogg-Shafarevich formula and the functional equation. His methods also use facts about the structure of the set $\mathrm{GL}_{2}(F) \backslash \mathrm{GL}_{2}(\mathbb{A})$ specific to the rational function field, and hence it is impossible to generalise his approach in order to show Corollary 7.7 in general.

\section{Elliptic curves with positive Manin constant}

Definition 8.1. Fix a positive integer $n$ that is not divisible by $p$. Let $E_{n}$ be the elliptic curve over $F=\mathbb{F}_{q}(T)$ (where $q=p^{d}$ ) with plane cubic model

$$
y^{2}+x y=x^{3}-T^{n} .
$$

Straightforward calculation shows that $j(E)^{-1}=T^{n}\left(1-2^{4} 3^{3} T^{n}\right)$. Hence $E$ is not isotrivial. Let $\Delta_{E_{n}}$ denote the discriminant of a relatively minimal elliptic surface $\mathscr{E}_{n} \rightarrow \mathscr{C}$ whose generic fibre is $E_{n}$. The degree of $\Delta_{E_{n}}$ can be easily computed from [Ulmer 2002, 2.2 and 2.3]. In particular, when $p \geq 5$, it follows at once from these results and [Silverman 1994, Table 4.1] that $\operatorname{deg}\left(\Delta_{E_{n}}\right)=12\lceil n / 6\rceil$.

Definition 8.2. Let $F_{n}$ be the Fermat surface of degree $n$ over $\mathbb{F}_{q}$, that is, the hypersurface in $\mathbb{P}_{\mathbb{F}_{q}}^{3}$ defined by the equation

$$
x_{0}^{n}+x_{1}^{n}+x_{2}^{n}+x_{3}^{n}=0 \text {. }
$$

Let $\mu_{n}$ denote the group of $n$-th roots of unity in $\overline{\mathbb{F}}_{p}$. Let $G$ be the quotient of $\mu_{n}^{4}$ modulo the diagonally embedded copy of $\mu_{n}$. For every $\underline{z}=\left(\zeta_{0}, \zeta_{1}, \zeta_{2}, \zeta_{3}\right) \in \mu_{n}^{4}$, let $\left[\zeta_{0}, \zeta_{1}, \zeta_{2}, \zeta_{3}\right]$ denote the image of $\underline{z}$ under the quotient map $\mu_{n}^{4} \rightarrow G$. Then the group scheme $G$ acts on $F_{n}$ and the action on the level of points is given by the rule

$$
\left[\zeta_{0}, \zeta_{1}, \zeta_{2}, \zeta_{3}\right] \cdot\left[x_{0}: x_{1}: x_{2}: x_{3}\right]=\left[\zeta_{0} x_{0}: \zeta_{1} x_{1}: \zeta_{2} x_{2}: \zeta_{3} x_{3}\right]
$$

Fix a primitive $n$-th root of unity $\zeta \in \overline{\mathbb{F}}_{p}$ and let $\Gamma \subset G$ be the subgroup generated by $\left[\zeta^{2}, \zeta, 1,1\right]$ and $\left[1, \zeta, \zeta^{3}, 1\right]$. Since a subgroup-scheme $\Gamma$ is defined over $\mathbb{F}_{q}$, the quotient surface $F_{n} / \Gamma$ is defined over $\mathbb{F}_{q}$ too.

Theorem 8.3. The surfaces $\mathscr{E}_{n}$ and $F_{n} / \Gamma$ are birationally equivalent.

Proof. The proof of this claim can be found in [Ulmer 2002, pp. 298-301]. 
Notation 8.4. Let $\mathbb{O}_{p}$ denote the ring of integers of $\overline{\mathbb{Q}}_{p}$, and let $\mathfrak{p}$ be its maximal ideal. We view all finite fields of characteristic $p$ as subfields of $\mathbb{O}_{p} / \mathfrak{p}$, which is an algebraic closure of $\mathbb{F}_{p}$. Reduction modulo $\mathfrak{p}$ induces an isomorphism between the group of all roots of unity of order prime to $p$ in $\mathbb{O}_{p}$ and the multiplicative group of $\mathbb{O}_{p} / \mathfrak{p}$. We let $\alpha:\left(\mathbb{O}_{p} / \mathfrak{p}\right)^{*} \rightarrow \overline{\mathbb{Q}}_{p}^{*}$ denote the inverse of this isomorphism. We will use the same letter $\alpha$ for the restriction to any finite field $\mathbb{F}_{q}^{*}$.

Definition 8.5. Fix a nontrivial character $\psi_{0}: \mathbb{F}_{p} \rightarrow \overline{\mathbb{Q}}_{p}^{*}$, and for each finite extension $\mathbb{F}_{p^{m}}$ of $\mathbb{F}_{p}$, let $\psi: \mathbb{F}_{p^{m}} \rightarrow \overline{\mathbb{Q}}_{p}^{*}$ be defined by $\psi=\psi_{0} \circ \operatorname{Tr}_{\mathbb{F}_{p^{m}} \mid \mathbb{F}_{p}}$. If $\chi: \mathbb{F}_{p^{m}}^{*} \rightarrow \overline{\mathbb{Q}}_{p}^{*}$ is a nontrivial character, we define the corresponding Gauss sum by

$$
g(\chi, \psi)=-\sum_{x \in \mathbb{F}_{p^{m}}^{*}} \chi(x) \psi(x)
$$

If $\chi_{1}, \ldots, \chi_{r}$ are characters $\mathbb{F}_{p^{m}}^{*} \rightarrow \overline{\mathbb{Q}}_{p}^{*}$, not all trivial, such that the product $\chi_{1} \cdots \chi_{r}$ is trivial, we define the Jacobi sum $J\left(\chi_{1}, \ldots, \chi_{r}\right)$ by

$$
J\left(\chi_{1}, \ldots, \chi_{r}\right)=\left\{\begin{array}{cl}
\frac{(-1)^{r}}{p^{m}} \prod_{i=1}^{r} g\left(\chi_{i}, \psi\right), & \text { if all } \chi_{i} \text { are nontrivial, } \\
0, & \text { otherwise. }
\end{array}\right.
$$

Theorem 8.6 (Stickelberger). For any $1 \leq k \leq p^{m}-2$, we have

$$
v_{p}\left(g\left(\alpha^{-k}, \psi\right)\right)=s(k) /(p-1),
$$

where if $k=k_{0}+p k_{1}+\cdots+p^{m-1} k_{m-1}$ is the $p$-adic expansion of the integer $k$, we define $s(k)=k_{0}+k_{1}+\cdots+k_{m-1}$.

Proof. This is the second claim of [Lang 1994, Theorem 9].

Definition 8.7. Let $G$ be the group that we introduced in Definition 8.2. Let $\widehat{G}$ denote the group of characters $G$ with values in $\overline{\mathbb{Q}}_{p}$. Using the character $\alpha$ : $\left(\mathbb{O}_{p} / \mathfrak{p}\right)^{*} \rightarrow \overline{\mathbb{Q}}_{p}^{*}$ we can identify $\widehat{G}$ with

$$
\left\{a=\left(a_{0}, a_{1}, a_{2}, a_{3}\right) \in(\mathbb{Z} / n \mathbb{Z})^{4} \mid \sum a_{i}=0\right\},
$$

where the duality pairing $G \times \widehat{G} \rightarrow \overline{\mathbb{Q}}_{p}^{*}$ is

$$
a(z)=\left\langle\left(a_{0}, a_{1}, a_{2}, a_{3}\right),\left[\zeta_{0}, \zeta_{1}, \zeta_{2}, \zeta_{3}\right]\right\rangle=\prod_{i=0}^{3} \alpha\left(\zeta_{i}\right)^{a_{i}} .
$$

For every $a \in \widehat{G}$, let $u(a)$ denote the smallest positive integer such that $q^{u(a)} a=$ $a$. For every nonzero $a=\left(a_{0}, a_{1}, a_{2}, a_{3}\right) \in \widehat{G}$, define the Jacobi sum $J(a)$ as follows: let $\chi_{i}: \mathbb{F}_{q^{u(a)}}^{*} \rightarrow \overline{\mathbb{Q}}_{p}$ be defined as $\chi_{i}=\alpha^{\left(\left(q^{u(a)}-1\right) / d\right) a_{i}}$, and set $J(a)=$ $J\left(\chi_{0}, \ldots, \chi_{3}\right)$. Note that $J(q a)=J(a)$. By convention, we set $J(0)=q$. Let 
$\Gamma^{\perp} \subset \widehat{G}$ be the cyclic subgroup of order $n$ generated by $(3,-6,2,1)$ and let

$$
\widehat{G}^{\prime}=\left\{a=\left(a_{0}, \ldots, a_{3}\right) \in \widehat{G} \mid a=0 \text { or } a_{i} \neq 0 \text { for } i=0, \ldots 3\right\} .
$$

As in Notation 6.5, for every $\operatorname{Spec}\left(\mathbb{F}_{q}\right)$-scheme $S$ let $\bar{S}$ denote again its base change to $\operatorname{Spec}\left(\overline{\mathbb{F}}_{p}\right)$ and let $F: \bar{S} \rightarrow \bar{S}$ denote the Frobenius relative to $\mathbb{F}_{q}$ for every such $S$. Choose a prime $l \neq p$ and fix an isomorphism $v: \overline{\mathbb{Q}}_{l} \rightarrow \overline{\mathbb{Q}}_{p}$. We will identify $\overline{\mathbb{Q}}_{l}$ with $\overline{\mathbb{Q}}_{p}$ via $v$.

Theorem 8.8. Let $A_{1}, \ldots, A_{k}$ be the orbits of multiplication by $q$ on $\Gamma^{\perp} \cap \widehat{G}^{\prime}$ and choose $a_{i} \in A_{i}$. Then

$$
\operatorname{det}\left(1-F^{*} t \mid H^{2}\left(\overline{F_{n} / \Gamma}, \overline{\mathbb{Q}}_{l}\right)\right)=\prod_{i=1}^{k}\left(1-J\left(a_{i}\right) t^{u\left(a_{i}\right)}\right) .
$$

Proof. This is [Ulmer 2002, Corollary 7.7].

Definition 8.9. As in Notation 3.1 , pick an isomorphism $\imath: \overline{\mathbb{Q}}_{l} \rightarrow \mathbb{C}$ and identify $\overline{\mathbb{Q}}_{l}$ with $\mathbb{C}$ via $l$ in all that follows. Let $|\cdot|_{\infty}$ denote the usual archimedean absolute value on $\mathbb{C}$. For every $\alpha \in \mathbb{R}$, finite dimensional $\overline{\mathbb{Q}}_{l}$-vector space $V$ and $\overline{\mathbb{Q}}_{l}$-linear endomorphism $\Psi: V \rightarrow V$ we say that the pair $(V, \Psi)$ has weights at most $\alpha$ if for every eigenvalue $\lambda$ of $\Psi$ we have $|\lambda|_{\infty} \leq|q|^{\alpha}$. Moreover we say that $(V, \Psi)$ has slope $\alpha$ if for every $\lambda$ as above $v_{q}(\lambda)=\alpha$. For every $\alpha \in \mathbb{R}, k \in \mathbb{N}$ and scheme $X$ of finite type over $\operatorname{Spec}\left(\mathbb{F}_{q}\right)$ we say that $X$ has weights at most $\alpha$ in dimension $k$ if the pair $\left(H^{k}\left(\bar{X}, \overline{\mathbb{Q}}_{l}\right), F^{*}\right)$ has weights at most $\alpha$. Similarly we say that $X$ has slope $\alpha$ in dimension $k$ if the pair $\left(H^{k}\left(\bar{X}, \overline{\mathbb{Q}}_{l}\right), F^{*}\right)$ has slope $\alpha$.

Now let $C$ be a curve over $\mathbb{F}_{q}$, that is, a one-dimensional (but necessarily equidimensional) variety defined over $\mathbb{F}_{q}$.

Lemma 8.10. The curve $C$ has weights at most 1 in dimension 1 , and the curve $C$ has slope 1 in dimension 2.

Proof. Let $U$ be a smooth dense open subscheme of $C$ and let $i: U \rightarrow C$ be the inclusion map. Let $S$ be the reduced closed subscheme of $C$ whose underlying set is the complement of $U$ and let $j: S \rightarrow C$ be the inclusion map. Then there is a cohomological long exact sequence

$$
\begin{aligned}
H^{1}\left(\bar{C}, i_{!}\left(\overline{\mathbb{Q}}_{l}\right)\right) \stackrel{\alpha}{\longrightarrow} H^{1}\left(\bar{C}, \overline{\mathbb{Q}}_{l}\right) \longrightarrow H^{1}\left(\bar{C}, j_{*}\left(\overline{\mathbb{Q}}_{l}\right)\right) \longrightarrow \\
\cdots \longrightarrow H^{2}\left(\bar{C}, i_{!}\left(\overline{\mathbb{Q}}_{l}\right)\right) \stackrel{\beta}{\longrightarrow} H^{2}\left(\bar{C}, \overline{\mathbb{Q}}_{l}\right) \longrightarrow H^{2}\left(\bar{C}, j_{*}\left(\overline{\mathbb{Q}}_{l}\right)\right) .
\end{aligned}
$$

Because $j_{*}\left(\mathbb{Q}_{l}\right)$ is the direct sum of skyscraper sheaves, it is acyclic. Hence the map $\alpha$ is surjective and the map $\beta$ is an isomorphism. By the proper base change theorem, $H_{c}^{1}\left(\bar{U}, \overline{\mathbb{Q}}_{l}\right)=H^{1}\left(\bar{C}, i_{!}\left(\overline{\mathbb{Q}}_{l}\right)\right)$ so the pair $\left(H^{1}\left(\bar{C}, i_{!}\left(\overline{\mathbb{Q}}_{l}\right)\right), F^{*}\right)$ has weights at most 1 by Deligne's purity theorem. Similarly, $H_{c}^{2}\left(\bar{U}, \overline{\mathbb{Q}}_{l}\right)=H^{2}\left(\bar{C}, i_{!}\left(\overline{\mathbb{Q}}_{l}\right)\right)$ so 
the pair $\left(H^{2}\left(\bar{C}, i_{!}\left(\overline{\mathbb{Q}}_{l}\right), F^{*}\right)\right.$ has slope 1 by the duality theorem. Because the maps $\alpha$ and $\beta$ are $F^{*}$-equivariant, the claims are now clear.

Notation 8.11. Now let $X$ be a surface over $\mathbb{F}_{q}$, that is, a two-dimensional variety defined over $\mathbb{F}_{q}$. Let $U$ be a smooth dense open subscheme of $X$ and let $i: U \rightarrow X$ be the inclusion map. Then we have a map

$$
i_{*}: H_{c}^{2}\left(\bar{U}, \overline{\mathbb{Q}}_{l}\right) \longrightarrow H^{2}\left(\bar{X}, \overline{\mathbb{Q}}_{l}\right)
$$

that is the composition of the isomorphism $H_{c}^{2}\left(\bar{U}, \overline{\mathbb{Q}}_{l}\right)=H^{2}\left(\bar{X}, i_{!}\left(\overline{\mathbb{Q}}_{l}\right)\right)$ furnished by proper base change and the homomorphism $H^{2}\left(\bar{X}, i_{!}\left(\overline{\mathbb{Q}}_{l}\right)\right) \rightarrow H^{2}\left(\bar{X}, \overline{\mathbb{Q}}_{l}\right)$ induced by the inclusion $i_{!}\left(\overline{\mathbb{Q}}_{l}\right) \subseteq \overline{\mathbb{Q}}_{l}$.

Lemma 8.12. (i) The pair $\left(\operatorname{Ker}\left(i_{*}\right), F^{*}\right)$ has weights at most 1 .

(ii) The pair $\left(\operatorname{Coker}\left(i_{*}\right), F^{*}\right)$ has slope 1 .

Proof. Let $C$ be the reduced closed subscheme whose underlying set is the complement of $U$ and let $j: C \rightarrow X$ be the inclusion map. Then $C$ is a curve and there is a cohomological long exact sequence

$$
H^{1}\left(\bar{X}, j_{*}\left(\overline{\mathbb{Q}}_{l}\right)\right) \rightarrow H^{2}\left(\bar{X}, i_{!}\left(\overline{\mathbb{Q}}_{l}\right)\right) \rightarrow H^{2}\left(\bar{X}, \overline{\mathbb{Q}}_{l}\right) \rightarrow H^{2}\left(\bar{X}, j_{*}\left(\overline{\mathbb{Q}}_{l}\right)\right) .
$$

Because $H^{1}\left(\bar{X}, j_{*}\left(\overline{\mathbb{Q}}_{l}\right)\right)=H^{1}\left(\bar{C}, \overline{\mathbb{Q}}_{l}\right)$ and $H^{2}\left(\bar{X}, j_{*}\left(\overline{\mathbb{Q}}_{l}\right)\right)=H^{2}\left(\bar{C}, \overline{\mathbb{Q}}_{l}\right)$, the pair $\left(H^{1}\left(\bar{X}, j_{*}\left(\overline{\mathbb{Q}}_{l}\right)\right), F^{*}\right)$ has weights at most 1 and the pair $\left(H^{2}\left(\bar{X}, j_{*}\left(\overline{\mathbb{Q}}_{l}\right)\right), F^{*}\right)$ has slope 1 by Lemma 8.10. The claims are now clear.

Proposition 8.13. Let $X_{1}$ and $X_{2}$ be two birationally equivalent geometrically irreducible projective surfaces over $\operatorname{Spec}\left(\mathbb{F}_{q}\right)$. Assume that both $X_{1}$ and $X_{2}$ are pure of weight 2 in dimension 2. Then

$$
l_{q}\left(\operatorname{det}\left(1-F^{*} t \mid H^{2}\left(\bar{X}_{1}, \overline{\mathbb{Q}}_{l}\right)\right)\right)=l_{q}\left(\operatorname{det}\left(1-F^{*} t \mid H^{2}\left(\bar{X}_{2}, \overline{\mathbb{Q}}_{l}\right)\right)\right) .
$$

Proof. Let $U$ be a smooth, two-dimensional scheme over $\operatorname{Spec}\left(\mathbb{F}_{q}\right)$ such that there are open immersions $i_{1}: U \rightarrow X_{1}$ and $i_{2}: U \rightarrow X_{1}$. Then

$$
l_{q}\left(\operatorname{det}\left(1-F^{*} t \mid \operatorname{Im}\left(i_{k *}\right)\right)=l_{q}\left(\operatorname{det}\left(1-F^{*} t \mid H^{2}\left(\bar{X}_{k}, \overline{\mathbb{Q}}_{l}\right)\right)\right)\right.
$$

for $k=1,2$, using the notation of 8.11 , by Lemma 8.12(ii). Let $V$ denote the largest $F^{*}$-invariant $\overline{\mathbb{Q}}_{l}$-linear subspace of $H_{c}^{2}\left(\bar{U}, \overline{\mathbb{Q}}_{l}\right)$ that has weights at most 1. Because $H^{2}\left(\bar{X}_{k}, \overline{\mathbb{Q}}_{l}\right)$ is pure of weight 2 we have $V \subseteq \operatorname{Ker}\left(i_{k *}\right)$ for $k=1$, 2. But $V \supseteq \operatorname{Ker}\left(i_{k *}\right)$ by Lemma 8.12(i). Hence

$$
\operatorname{det}\left(1-F^{*} t \mid \operatorname{Im}\left(i_{1 *}\right)\right)=\operatorname{det}\left(1-F^{*} t \mid H_{c}^{2}\left(\bar{U}, \overline{\mathbb{Q}}_{l}\right) / V\right)=\operatorname{det}\left(1-F^{*} t \mid \operatorname{Im}\left(i_{2 *}\right)\right),
$$

so the claim is clear.

Now assume that $q=p$. 
Theorem 8.14. Let $p$ be a prime number and let $n$ be a positive integer as above. Assume that $n \mid p-1$ and $6 \mid n$. Then $E_{n}$ is not isotrivial and

$$
m\left(E_{n}\right)=\frac{1}{6} n-1=\frac{1}{12} \operatorname{deg}\left(\Delta_{E_{n}}\right)-1 .
$$

Proof. Let $\underline{a}=(-3,6,-2,-1) \in \widehat{G}$. By our assumption for every $k=0,1, \ldots, n-$ 1 we have $k \underline{a} \in \widehat{G}^{\prime}$ if and only if $k \neq n / 6, n / 3, n / 2,2 n / 3$ or $5 n / 6$. Because of our assumption $n \mid p-1$ every orbit of multiplication by $p$ on $\Gamma^{\perp} \cap \widehat{G}^{\prime}$ consists of one element. Hence

$$
\operatorname{det}\left(1-F^{*} t \mid H^{2}\left(\overline{F_{n} / \Gamma}, \overline{\mathbb{Q}}_{l}\right)\right)=\prod_{\substack{0 \leq k \leq n-1 ; \\ k \neq n / 6, n / 3, n / 2, 2 n / 3,5 n / 6}}(1-J(k \underline{a}) t)
$$

by Theorem 8.8. Gauss sums are Weil numbers of weight 1 , and hence the reciprocal roots of the polynomial in (25) are Weil numbers of weight 2 . The surface $\mathscr{E}_{n}$ is smooth, so it is pure of weight 2 in dimension 2 by Deligne's purity theorem. Hence by Theorem 8.3 and Proposition 8.13 , we have

$$
l_{q}\left(L\left(E_{n}, t\right)\right)=l_{q}\left(\operatorname{det}\left(1-F^{*} t \mid H^{2}\left(\overline{\mathscr{E}}_{n}, \overline{\mathbb{Q}}_{l}\right)\right)\right)=l_{q}\left(\operatorname{det}\left(1-F^{*} t \mid H^{2}\left(\bar{F}_{n} / \Gamma, \overline{\mathbb{Q}}_{l}\right)\right)\right) .
$$

For every $k=1,2, \ldots, n / 6-1$, we have

$$
\begin{aligned}
& v_{p}(J(k \underline{a}))= \\
& v_{p}\left(g\left(\alpha^{-3 k(p-1) / n}, \psi\right) g\left(\alpha^{6 k(p-1) / n-p+1}, \psi\right) g\left(\alpha^{-2 k(p-1) / n}, \psi\right) g\left(\alpha^{-k(p-1) / n}, \psi\right)\right)-1 \\
& =\frac{3 k}{n}+1-\frac{6 k}{n}+\frac{2 k}{n}+\frac{k}{n}-1=0
\end{aligned}
$$

by Theorem 8.6, since every exponent of $\alpha^{-1}$ in the equation above is a positive integer strictly less than $p$. Hence by Proposition 4.5 and the above,

$$
m\left(E_{n}\right) \geq l_{q}\left(L\left(E_{n}, t\right)\right) \geq \frac{1}{6} n-1 .
$$

Because $6 \mid p-1$ by our assumptions, we have $p \geq 7$. Hence

$$
m\left(E_{n}\right) \leq \frac{1}{12} \operatorname{deg}\left(\Delta_{E_{n}}\right)-1=\frac{1}{6} n-1
$$

by Theorem 6.9 and by our remark at the end of Definition 8.1 .

\section{Strong Weil curves}

Definition 9.1. Fix now a closed point $\infty$ of $\mathscr{C}$ and let $A$ denote the ring of rational functions on $\mathscr{C}$ regular away from $\infty$ as in the introduction. For any nonzero ideal $\mathfrak{m}$ of $A$, an irreducible affine algebraic curve $Y_{0}(\mathfrak{m})$ is defined over $F$, the Drinfeld modular curve parametrising Drinfeld $A$-modules of rank 2 of generic characteristic with Hecke level $\mathfrak{m}$-structure. There is a unique nonsingular projective curve 
$X_{0}(\mathfrak{m})$ over $F$ that contains $Y_{0}(\mathfrak{m})$ as a dense open subvariety. Let $J_{0}(\mathfrak{m})$ denote the Jacobian of the curve $X_{0}(\mathfrak{m})$. The ideals of $A$ and the effective divisors on $\mathscr{b}$ whose support does not contain $\infty$ are in a natural one-to-one correspondence, and we will not distinguish them in what follows. Let $E$ be an elliptic curve defined over $F$ that has split multiplicative reduction at $\infty$. Then its conductor is of the form $\mathfrak{m} \infty$, where $\mathfrak{m}$ is an ideal of $A$. By the function field analogue of the Taniyama-Weil conjecture, there is a nonconstant map $\pi: X_{0}(\mathfrak{m}) \rightarrow E$ defined over $F$. For any map $h: X_{0}(\mathfrak{m}) \rightarrow C$, where $C$ is an elliptic curve, let $h_{*}: J_{0}(\mathfrak{m}) \rightarrow C$ and $h^{*}: C \rightarrow J_{0}(\mathfrak{m})$ denote the maps induced by the Albanese and the Picard functorialities.

Theorem 9.2. The following are equivalent:

(i) The kernel of the map $\pi_{*}: J_{0}(\mathfrak{m}) \rightarrow E$ is an abelian variety.

(ii) The map $\pi^{*}: E \rightarrow J_{0}(\mathfrak{m})$ is a closed immersion.

(iii) The degree of $\pi$ is minimal among all nondegenerate maps $\rho: X_{0}(\mathfrak{m}) \rightarrow E^{\prime}$, where $E^{\prime}$ is any elliptic curve isogenous to $E$ over $F$.

Proof. This result is well known in the mathematical folklore, but it is difficult to track down a proof - the standard reference, [Mazur 1973, Lemme 3], only shows the equivalence of (i) and (iii). Our excuse for giving a full proof other than that is that we consider the more delicate case of positive characteristic. First assume that (i) holds. By the multiplicity 1 theorem for the action of the Hecke algebra on $J_{0}(\mathfrak{m})$, the abelian variety $\operatorname{Ker}\left(\pi_{*}\right)$ has no quotient isogenous to $E$, so for every nondegenerate map $\rho: X_{0}(\mathfrak{m}) \rightarrow E^{\prime}$, where $E^{\prime}$ is any elliptic curve isogenous to $E$ over $F$, the kernel of $\rho_{*}$ must contain $\operatorname{Ker}\left(\pi_{*}\right)$. Hence the map $\rho$ factors through $\pi$, and in particular its degree is at least as big as that of $\pi$. On the other hand, if (iii) holds, then $\operatorname{Ker}\left(\pi_{*}\right)$ contains the reduction of the connected component of its identity element as a closed subgroup-scheme. The quotient $E^{\prime}$ of $J_{0}(\mathfrak{m})$ by the latter is an elliptic curve isogenous to $E$; hence the degree of the corresponding map $\rho: X_{0}(\mathfrak{m}) \rightarrow E^{\prime}$ is at least as big as $\operatorname{deg}(\pi)$. But $\pi$ factors through $\rho$, so they must be equal. Note that the map $\pi^{*}$ is just the dual of the morphism $\pi_{*}: J_{0}(\mathfrak{m}) \rightarrow E$ of the principally polarised abelian varieties. The equivalence of (i) and (ii), and therefore the theorem itself, now follows from the lemma below.

Lemma 9.3. Let $\phi: A \rightarrow B$ be a surjective homomorphism of abelian varieties and let $\phi^{\vee}: B^{\vee} \rightarrow A^{\vee}$ be its dual. Then the following are equivalent:

(i) The kernel of $\phi$ is an abelian variety.

(ii) The map $\phi^{\vee}$ is a closed immersion.

Proof. This proof was explained to me by Laurent Fargues. For any $S$-scheme $T$ let $T$ also denote the sheaf represented by $T$ on the fppf topology on $S$. Attached 
to the short exact sequence

$$
0 \longrightarrow \operatorname{Ker}(\phi) \longrightarrow A \stackrel{\phi}{\longrightarrow} B \longrightarrow 0
$$

of sheaves on the fppf topology, there is a cohomological exact sequence

$$
\operatorname{Hom}\left(A, \mathbb{G}_{m}\right) \longrightarrow \operatorname{Hom}\left(\operatorname{Ker}(\phi), \mathbb{G}_{m}\right) \longrightarrow \operatorname{Ext}^{1}\left(B, \mathbb{G}_{m}\right) \stackrel{\phi^{\vee}}{\longrightarrow} \operatorname{Ext}^{1}\left(A, \mathbb{G}_{m}\right) .
$$

By a theorem of Grothendieck, for any abelian scheme $C$ the sheaf $\operatorname{Ext}^{1}\left(C, \mathbb{G}_{m}\right)$ is represented by the dual of $C$ and for any morphism $\phi: A \rightarrow B$ of abelian varieties the induced map $\operatorname{Ext}^{1}\left(B, \mathbb{G}_{m}\right) \rightarrow \operatorname{Ext}^{1}\left(A, \mathbb{G}_{m}\right)$ is the dual of $\phi$, as the notation above indicates. Moreover the sheaf $\operatorname{Hom}\left(C, \mathbb{G}_{m}\right)$ is trivial for any abelian scheme $C$, and hence $\phi^{\vee}$ is an immersion if and only if $\operatorname{Hom}\left(\operatorname{Ker}(\phi), \mathbb{G}_{m}\right)$ is trivial. Let $\operatorname{Ker}(\phi)_{0}$ denote the reduced group scheme associated to the connected component of $\operatorname{Ker}(\phi)$, considered as a closed subgroup-scheme. It is an abelian subscheme of $\operatorname{Ker}(\phi)$ such that the quotient $G=\operatorname{Ker}(\phi) / \operatorname{Ker}(\phi)_{0}$ is a finite, flat group scheme. By looking at the cohomological exact sequence attached to the short exact sequence

$$
0 \longrightarrow \operatorname{Ker}(\phi)_{0} \longrightarrow \operatorname{Ker}(\phi) \longrightarrow G \longrightarrow 0
$$

of sheaves on the fppf topology, we get that $\operatorname{Hom}\left(\operatorname{Ker}(\phi), \mathbb{G}_{m}\right)=\operatorname{Hom}\left(G, \mathbb{G}_{m}\right)$. The sheaf $\operatorname{Hom}\left(G, \mathbb{G}_{m}\right)$ is represented by the Cartier dual of the group scheme $G$, so it is trivial if and only if $G$ is trivial.

Definition 9.4. If the equivalent conditions of Lemma 9.3 hold, then we say that $E$ is a strong Weil curve and the modular parametrisation $\pi: X_{0}(\mathfrak{m}) \rightarrow E$ is optimal. By the proof above, it is clear that up to isomorphism $E$ is unique in its isogeny class and there is only one strong Weil map parametrising $E$. On the other hand, by property (i), the quotient of $J_{0}(\mathfrak{m})$ by the reduced group scheme associated to the connected component of the kernel of the map $\pi_{*}: J_{0}(\mathfrak{m}) \rightarrow E$ induced by any modular parametrisation $\pi: X_{0}(\mathfrak{m}) \rightarrow E$ is a strong Weil curve. Hence there is a strong Weil curve in the isogeny class of every elliptic curve having split multiplicative reduction at $\infty$.

\section{Applications to the degree conjecture}

Definition 10.1. For any graph $G$, let $\mathscr{V}(G)$ and $\mathscr{E}(G)$ denote its set of vertices and edges, respectively. Let $R$ be a commutative group and let $G$ be a locally finite oriented graph. In this paper we will assume that every oriented graph $G$ is equipped with an involution $:: \mathscr{E}(G) \rightarrow \mathscr{E}(G)$ such that for each edge $e \in \mathscr{E}(G)$, the original and terminal vertices of the edge $\bar{e} \in \mathscr{E}(G)$ are the terminal and original vertices of $e$, respectively. The edge $\bar{e}$ is called the edge $e$ with reversed orientation. If for each edge $e \in \mathscr{E}(G)$ there is exactly one edge $\bar{e} \in \mathscr{E}(G)$ whose original and terminal vertices are the terminal and original vertices of $e$, then there is a 
unique involution of this type. The Bruhat-Tits tree $\mathscr{T}$ is such a graph. A function $\phi: \mathscr{E}(G) \rightarrow R$ is called a harmonic $R$-valued cochain if it satisfies the following conditions:

- $\phi(e)+\phi(\bar{e})=0$ for all $e \in \mathscr{E}(G)$.

- If for an edge $e$ we introduce the notation $o(e)$ and $t(e)$ for its original and terminal vertex respectively,

$$
\sum_{\substack{e \in \mathscr{E}(G) \\ o(e)=v}} \phi(e)=0 \quad \text { for all } v \in \mathscr{V}(G) .
$$

We denote by $H(G, R)$ the group of $R$-valued harmonic cochains on $G$.

Definition 10.2. Let $Y \subset F^{2}$ be an $A$-lattice, that is, a projective $A$-submodule of rank 2. Let $\Gamma(Y)$ denote the $F$-linear automorphisms of $F^{2}$ leaving $Y$ invariant, and for every ideal $\mathfrak{a} \triangleleft A$, let $\Gamma(Y, \mathfrak{a}) \leq \Gamma(Y)$ denote the subgroup of those elements that induce the identity on the quotient $A$-module $Y / \mathfrak{a} Y$. We say that a subgroup $\Gamma$ of $\mathrm{GL}_{2}(F)$ is arithmetic if there is an $A$-lattice $Y$ and an ideal $\mathfrak{a}$ such that $\Gamma$ is contained in $\Gamma(Y)$ and it contains $\Gamma(Y, \mathfrak{a})$. Let $\Gamma$ be an arithmetic subgroup of $\mathrm{GL}_{2}(F)$ and let $F_{\infty}$ denote the completion of $F$ with respect to the valuation corresponding to $\infty$. As a subgroup of $\mathrm{GL}_{2}\left(F_{\infty}\right)$, the arithmetic group $\Gamma$ acts on the Bruhat-Tits tree associated to $\mathrm{PGL}_{2}\left(F_{\infty}\right)$ on the left, which we will denote by $\mathscr{T}$. For any abelian group $M$, let $H_{!}(\mathcal{T}, M)^{\Gamma}$ denote the group of those $\Gamma$-invariant, $M$-valued harmonic cochains on $\mathscr{T}$ that has finite support as a function on the edges of the quotient graph $\Gamma \backslash \mathcal{T}$.

Definition 10.3. Next we define the first homology group $H_{1}(\Gamma \backslash \mathcal{T}, \mathbb{Z})$, which has several descriptions. It may be defined as the first topological homology group of the CW-complex attached to $\Gamma \backslash \mathcal{T}$ with integral coefficients. It is also canonically isomorphic to the abelianization of the quotient $\Gamma^{*}=\Gamma / \Gamma_{f}$, where $\Gamma_{f}$ is the normal subgroup of $\Gamma$ generated by the elements of finite order. We will use a third, purely combinatorial description, since it is the most convenient for our purposes. Recall that a path on an oriented graph $G$ is a sequence of edges $e_{1}, e_{2}, \ldots, e_{n} \in \mathscr{E}(G)$ such that $t\left(e_{i}\right)=o\left(e_{i+1}\right)$ for $i=1,2, \ldots, n-1$. The path is closed if the equality $t\left(e_{n}\right)=o\left(e_{1}\right)$ holds, too. For each edge $e \in \mathscr{E}(G)$, let $i_{e}: \mathscr{E}(G) \rightarrow \mathbb{Z}$ denote the unique function such that

$$
i_{e}(f)=\left\{\begin{aligned}
+1 & \text { if } f=e \\
-1 & \text { if } f=\bar{e} \\
0 & \text { otherwise }
\end{aligned}\right.
$$

To any closed path $e_{1}, e_{2}, \ldots, e_{n}$ we associate the function $\sum_{i=1}^{n} i_{e_{i}}$. We define $H_{1}(G, \mathbb{Z})$ as the abelian group of $\mathbb{Z}$-valued functions on $\mathscr{E}(G)$ generated by these 
functions. Let us return to the special case $G=\Gamma \backslash \mathscr{T}$. Let $z(\Gamma)$ denote the cardinality of the center of $\Gamma$ and let $\Gamma_{e}$ be the stabiliser of the edge $e \in \mathscr{E}(\mathscr{T})$ in $\Gamma$. Let us quickly recall why $\Gamma_{e}$ is finite. Let $v: \mathrm{GL}_{2}\left(F_{\infty}\right) \rightarrow \mathbb{Z}$ be the composition of the determinant and the valuation, and let $\mathrm{GL}_{2}\left(F_{\infty}\right)_{0}$ denote its kernel. We claim that every arithmetic group $\Gamma$ lies in $\mathrm{GL}_{2}\left(F_{\infty}\right)_{0}$. Clearly it is enough to show this for $\Gamma(Y)$. The localisation of $Y$ at each prime of $A$ is a free module of rank 2, so the determinant of every element of $\Gamma(Y)$ is a unit at each prime of $A$, so it is in fact a unit of $A$. The latter are constants, so they have valuation zero. On the other hand, the stabiliser of $e$ in $\mathrm{GL}_{2}\left(F_{\infty}\right)_{0}$ is compact, so $\Gamma_{e}$ is finite as the intersection of a compact and a discrete group. We define

$$
j_{\Gamma}: H_{1}(\Gamma \backslash \mathscr{T}, \mathbb{Z}) \rightarrow H_{!}(\mathscr{T}, \mathbb{Z})^{\Gamma}
$$

as the map $\phi \mapsto \phi^{*}$ given by the rule $\phi^{*}(e)=\left|\Gamma_{e}\right| \phi(\tilde{e}) / z(\Gamma)$, where $\tilde{e}$ is the image of the edge $e$ in $\mathscr{E}(\Gamma \backslash \mathscr{T})$. It is easy to see that the homomorphism is well-defined, that is, $\phi^{*}$ is indeed a harmonic cochain.

Proposition 10.4. The homomorphism $j_{\Gamma}$ is injective with finite cokernel of exponent dividing $q^{\operatorname{deg}(\infty)}-1$.

Proof. The injectivity is trivial if the definition above is employed. Gekeler and Reversat [1996, Proposition 6.4.4] proved that the cokernel has index prime to the characteristic $p$, and if one uses this result, a careful reading of the proof of [Gekeler and Reversat 1996, Lemma 3.3.3] reveals that our proposition above has already been proved there. Here we reproduce their argument for the sake of the reader. Let $T$ be a maximal tree in the connected graph $\Gamma \backslash \mathcal{T}$ : such a tree exists by Zorn's lemma. By Serre's structure theorem, the graph $\Gamma \backslash \mathcal{T}$ is the union of a finite graph and finitely many ends, and hence the complement $\mathscr{E}(\Gamma \backslash \mathscr{T})-\mathscr{E}(T)$ is finite. Let $R=\left\{\tilde{e}_{1}, \ldots, \tilde{e}_{g}\right\}$ be a set of representatives of $\mathscr{E}(\Gamma \backslash \mathscr{T})-\mathscr{E}(T)$ modulo orientation, that is, for every edge $e \in \mathscr{E}(\Gamma \backslash \mathscr{T})-\mathscr{E}(T)$ let exactly one of the edges $e$ and $\bar{e}$ be listed in $R$. For each edge $\tilde{e}_{i} \in R$, let $c_{i}$ denote a closed path consisting of $\tilde{e}_{i}$ and a path connecting $t\left(e_{i}\right)$ with $o\left(e_{i}\right)$ in $T$. For any $\phi \in H_{!}(\mathscr{T}, \mathbb{Z})^{\Gamma}$, the function

$$
\phi-\sum_{i=1}^{g} \frac{\phi\left(e_{i}\right) z(\Gamma)}{\left|\Gamma_{e_{i}}\right|} j_{\Gamma}\left(c_{i}\right)
$$

vanishes identically outside of the maximal tree $T$, and hence vanishes everywhere. Therefore the cokernel of $j_{\Gamma}$ is annihilated by the smallest common multiple of the natural numbers $\left|\Gamma_{e_{i}}\right| / z(\Gamma)$. Since the torsion of the stabiliser of any edge $e \in \mathscr{E}(\mathscr{T})$ in the image of $\mathrm{GL}_{2}\left(F_{\infty}\right)_{0}$ in $P \mathrm{GL}_{2}\left(F_{\infty}\right)$ modulo its $p$-torsion group is a group of order $q^{\operatorname{deg}(\infty)}-1$, the claim is now clear. 
Notation 10.5. In the rest of the paper we assume that $E$ is a strong Weil curve and $\pi: X_{0}(\mathfrak{m}) \rightarrow E$ is optimal. Let $\Gamma$ be the arithmetic group:

$$
\left\{\left(\begin{array}{ll}
a & b \\
c & d
\end{array}\right) \in \mathrm{GL}_{2}(A) \mid c \in \mathfrak{m}\right\}
$$

One may associate to the strong Weil curve $E$ an element $v_{E} \in H_{!}(\mathcal{T}, \mathbb{Z})^{\Gamma}$ lying in the image of the map $j_{\Gamma}$ (for its definition see [Papikian 2007, 3.4]). The set $\mathscr{E}(\mathscr{T})$ can be identified with $\mathrm{GL}_{2}\left(F_{\infty}\right) / \Gamma_{\infty} Z\left(F_{\infty}\right)$, where $\Gamma_{\infty}$ is the Iwahori subgroup:

$$
\Gamma_{\infty}=\left\{\left(\begin{array}{ll}
a & b \\
c & d
\end{array}\right) \in \mathrm{GL}_{2}\left(\mathrm{O}_{\infty}\right) \mid \infty(c)>0\right\}
$$

There is a natural map

$$
h: \mathscr{E}(\mathscr{T}) \rightarrow \mathrm{GL}_{2}(F) \backslash \mathrm{GL}_{2}(\mathbb{A}) / \mathbb{K}_{0}(\mathfrak{m} \infty) Z(\mathbb{A})
$$

that for $g \in \mathrm{GL}_{2}\left(F_{\infty}\right)$ maps the left $\Gamma_{\infty} Z\left(F_{\infty}\right)$-coset of $g$ to the double coset $\mathrm{GL}_{2}(F) \mathbf{g} K_{0}(\mathfrak{m} \infty) Z(\mathbb{A})$ of the unique element $\mathbf{g} \in \mathrm{GL}_{2}(\mathbb{A})$ such that for every $x \in|\mathscr{C}|$, the $x$-th component of $\mathbf{g}$ is $g$ if $x$ is $\infty$, and 1 otherwise. By [Gekeler and Reversat 1996, 9.1], the function $v_{E}$ lies in the $\mathbb{Q}$-module $\mathbb{Q}\left(\psi_{E} \circ h\right)$ spanned by $\psi_{E} \circ h$. Let $\tilde{c}(E)$ be the unique nonnegative number such that $v_{E}=\tilde{c}(E) \psi_{E} \circ h$. By definition, $v_{E}$ generates the $\mathbb{Z}$-module $\operatorname{Im}\left(j_{\Gamma}\right) \cap \mathbb{Q}\left(\psi_{E} \circ h\right)$. Hence Proposition 10.4 has the following immediate corollary:

Corollary 10.6. $\tilde{c}(E) \leq\left(q^{\operatorname{deg}(\infty)}-1\right) c(E)^{-1}$.

Using the Riemann hypothesis for the $L$-function $L\left(\operatorname{Symm}^{2}(E), t\right)$ of the second symmetric square of the Galois representation $H^{1}\left(E_{\bar{F}}, \mathbb{Q}_{l}\right)$, Papikian [2007, Theorem 4.6 and Proposition 1.3] deduces the following from his main formula for the degree of modular parametrisations of elliptic curves:

Theorem 10.7. Assume that $\mathfrak{m}$ is square-free. Then

$$
\operatorname{deg}(\pi)<\tilde{c}(E)^{2} \cdot q^{14 g+\operatorname{deg}(\infty)+5} \cdot q^{\operatorname{deg}(\mathfrak{m})} \cdot \operatorname{deg}(\mathfrak{m})^{3} .
$$

Combining Corollary 7.7 with Corollary 10.6 and Theorem 10.7, we obtain the following result:

Theorem 10.8. Assume that $p$ does not divide the order of $\operatorname{Pic}_{0}(\mathscr{C})\left(\mathbb{F}_{q}\right)$, and that $\mathfrak{m}$ is square-free. Then

$$
\operatorname{deg}(\pi)<q^{18 g+4 \operatorname{deg}(\infty)+1} \cdot q^{2 \operatorname{deg}(\mathfrak{m})} \cdot \operatorname{deg}(\mathfrak{m})^{3} .
$$




\section{References}

[Chinburg 1994] T. Chinburg, "Galois structure of de Rham cohomology of tame covers of schemes", Ann. of Math. (2) 139:2 (1994), 443-490. MR 95h:11125a Zbl 0828.14007

[Deligne 1973] P. Deligne, "Les constantes des équations fonctionnelles des fonctions $L$ ", pp. 501597 in Modular functions of one variable (Antwerp, 1972), vol. II, edited by P. Deligne and W. Kuyk, Lecture Notes in Math 349, Springer, Berlin, 1973. MR 50 \#2128 Zbl 0271.14011

[Faber and van der Geer 2004] C. Faber and G. van der Geer, "Complete subvarieties of moduli spaces and the Prym map", J. Reine Angew. Math. 573 (2004), 117-137. MR 2005g:14054 Zbl 1075.14023

[Gekeler and Reversat 1996] E.-U. Gekeler and M. Reversat, "Jacobians of Drinfeld modular curves", J. Reine Angew. Math. 476 (1996), 27-93. MR 97f:11043 Zbl 0848.11029

[Goldfeld and Szpiro 1995] D. Goldfeld and L. Szpiro, "Bounds for the order of the Tate-Shafarevich group”, Compositio Math. 97:1-2 (1995), 71-87. MR 97a:11102 Zbl 0860.11032

[Harder 1974] G. Harder, "Chevalley groups over function fields and automorphic forms", Ann. of Math. (2) 100 (1974), 249-306. MR 58 \#27799 Zbl 0309.14041

[Illusie 1979] L. Illusie, "Complexe de de Rham-Witt et cohomologie cristalline", Ann. Sci. École Norm. Sup. (4) 12:4 (1979), 501-661. MR 82d:14013 Zbl 0436.14007

[Illusie 1990] L. Illusie, "Ordinarité des intersections complètes générales", pp. 376-405 in The Grothendieck Festschrift, vol. II, edited by P. Cartier et al., Progr. Math. 87, Birkhäuser, 1990. MR 93h:14015 Zbl 0728.14021

[Katz and Messing 1974] N. M. Katz and W. Messing, "Some consequences of the Riemann hypothesis for varieties over finite fields", Invent. Math. 23 (1974), 73-77. MR 48\#11117 Zbl 0275.14011

[Lang 1994] S. Lang, Algebraic number theory, 2nd ed., Grad. Texts in Math. 110, Springer, New York, 1994. MR 95f:11085 Zbl 0811.11001

[Laumon 1987] G. Laumon, “Transformation de Fourier, constantes d'équations fonctionnelles et conjecture de Weil”, Inst. Hautes Études Sci. Publ. Math. 65 (1987), 131-210. MR 88g:14019 Zbl 0641.14009

[Mazur 1972] B. Mazur, "Frobenius and the Hodge filtration", Bull. Amer. Math. Soc. 78 (1972), 653-667. MR 48 \#8507 Zbl 0258.14006

[Mazur 1973] B. Mazur, “Courbes elliptiques et symboles modulaires”, pp. 277-294 in Séminaire Bourbaki 1971/1972 (Exposé 414), Lecture Notes in Math. 317, Springer, Berlin, 1973. MR 55 \#2930 Zbl 0276.14012

[Miller 1972] L. Miller, "Curves with invertible Hasse-Witt matrix", Math. Ann. 197 (1972), 123127. MR 47 \#3399 Zbl 0235.14009

[Milne 1975] J. S. Milne, "On a conjecture of Artin and Tate", Ann. of Math. (2) 102:3 (1975), 517-533. MR 54 \#2659 Zbl 0343.14005

[Mumford 1969] D. Mumford, "Bi-extensions of formal groups", pp. 307-322 in Algebraic Geometry (Internat. Colloq.) (Bombay, 1968), Oxford Univ. Press, London, 1969. MR 41 \#1743 Zbl 0216.33101

[Norman and Oort 1980] P. Norman and F. Oort, "Moduli of abelian varieties", Ann. of Math. (2) 112:3 (1980), 413-439. MR 82h:14026 Zbl 0483.14010

[Papikian 2005] M. Papikian, "Pesenti-Szpiro inequality for optimal elliptic curves", J. Number Theory 114:2 (2005), 361-393. MR 2006f:11062 Zbl 1084.11027

[Papikian 2007] M. Papikian, "Analogue of the degree conjecture over function fields", Trans. Amer. Math. Soc. 359:7 (2007), 3483-3503. MR 2008d:11057 Zbl 05140897 
[Pesenti and Szpiro 2000] J. Pesenti and L. Szpiro, "Inégalité du discriminant pour les pinceaux elliptiques à réductions quelconques”, Compositio Math. 120:1 (2000), 83-117. MR 2001c:11063 Zbl 1021.11021

[Rück and Tipp 2000] H.-G. Rück and U. Tipp, "Heegner points and $L$-series of automorphic cusp forms of Drinfeld type”, Doc. Math. 5 (2000), 365-444. MR 2001i:11057 Zbl 1012.11039

[Seiler 1987] W. K. Seiler, "Global moduli for elliptic surfaces with a section”, Compositio Math. 62:2 (1987), 169-185. MR 88m:14027 Zbl 0624.14023

[Silverman 1986] J. H. Silverman, The arithmetic of elliptic curves, Graduate Texts in Mathematics 106, Springer, New York, 1986. MR 87g:11070 Zbl 0585.14026

[Silverman 1994] J. H. Silverman, Advanced topics in the arithmetic of elliptic curves, Graduate Texts in Mathematics 151, Springer, New York, 1994. MR 96b:11074 Zbl 0911.14015

[Tan 1993] K.-S. Tan, "Modular elements over function fields", J. Number Theory 45:3 (1993), 295-311. MR 95d:11158 Zbl 0802.11026

[Ulmer 2002] D. Ulmer, "Elliptic curves with large rank over function fields", Ann. of Math. (2) 155:1 (2002), 295-315. MR 2003b:11059 Zbl 1109.11314

Communicated by Richard Taylor

Received 2009-03-31 Revised 2009-12-02 Accepted 2009-12-31

a.pal@imperial.ac.uk

Department of Mathematics, Imperial College, 180 Queen's Gate, London, SW7 2AZ, United Kingdom 


\section{Algebra \& Number Theory}

www.jant.org

\section{EDITORS}

\section{MANAGING EDITOR}

Bjorn Poonen

Massachusetts Institute of Technology

Cambridge, USA

\author{
EDITORIAL BOARD CHAIR \\ David Eisenbud \\ University of California \\ Berkeley, USA
}

\section{BOARD OF EDITORS}

Georgia Benkart

Dave Benson

Richard E. Borcherds

John H. Coates

J-L. Colliot-Thélène

Brian D. Conrad

Hélène Esnault

Hubert Flenner

Edward Frenkel

Andrew Granville

Joseph Gubeladze

Ehud Hrushovski

Craig Huneke

Mikhail Kapranov

Yujiro Kawamata

János Kollár

Hendrik W. Lenstra

Yuri Manin

Barry Mazur
University of Wisconsin, Madison, USA

University of Aberdeen, Scotland

University of California, Berkeley, USA

University of Cambridge, UK

CNRS, Université Paris-Sud, France

University of Michigan, USA

Universität Duisburg-Essen, Germany

Ruhr-Universität, Germany

University of California, Berkeley, USA

Université de Montréal, Canada

San Francisco State University, USA

Hebrew University, Israel

University of Kansas, USA

Yale University, USA

University of Tokyo, Japan

Princeton University, USA

Universiteit Leiden, The Netherlands

Northwestern University, USA

Harvard University, USA
Susan Montgomery

Shigefumi Mori

Andrei Okounkov

Raman Parimala

Victor Reiner

Karl Rubin

Peter Sarnak

Michael Singer

Ronald Solomon

Vasudevan Srinivas

J. Toby Stafford

Bernd Sturmfels

Richard Taylor

Ravi Vakil

Michel van den Bergh

Marie-France Vignéras

Kei-Ichi Watanabe

Andrei Zelevinsky

Efim Zelmanov
University of Southern California, USA

RIMS, Kyoto University, Japan

Princeton University, USA

Emory University, USA

University of Minnesota, USA

University of California, Irvine, USA

Princeton University, USA

North Carolina State University, USA

Ohio State University, USA

Tata Inst. of Fund. Research, India

University of Michigan, USA

University of California, Berkeley, USA

Harvard University, USA

Stanford University, USA

Hasselt University, Belgium

Université Paris VII, France

Nihon University, Japan

Northeastern University, USA

University of California, San Diego, USA

\section{PRODUCTION}

ant@mathscipub.org

Silvio Levy, Scientific Editor

Andrew Levy, Production Editor

See inside back cover or www.jant.org for submission instructions.

The subscription price for 2010 is US \$140/year for the electronic version, and \$200/year (+\$30 shipping outside the US) for print and electronic. Subscriptions, requests for back issues from the last three years and changes of subscribers address should be sent to Mathematical Sciences Publishers, Department of Mathematics, University of California, Berkeley, CA 94720-3840, USA.

Algebra \& Number Theory (ISSN 1937-0652) at Mathematical Sciences Publishers, Department of Mathematics, University of California, Berkeley, CA 94720-3840 is published continuously online. Periodical rate postage paid at Berkeley, CA 94704, and additional mailing offices.

ANT peer review and production are managed by EditFLOW ${ }^{\mathrm{TM}}$ from Mathematical Sciences Publishers.

PUBLISHED BY

mathematical sciences publishers

http://www.mathscipub.org

A NON-PROFIT CORPORATION

Typeset in LATEX

Copyright $\odot 2010$ by Mathematical Sciences Publishers 


\section{Algebra \& Number Theory}

Volume $4 \quad$ No. $5 \quad 2010$

On the Spiegelungssatz for the 4-rank

ÉTIENNE FOUVRY and JÜRGEN KLÜNERS

The Manin constant of elliptic curves over function fields AMBRUS PÁL

Le problème de Bogomolov effectif sur les variétés abéliennes

AURÉLIEN GALATEAU

Transverse quiver Grassmannians and bases in affine cluster algebras GRÉGOIRE DUPONT

Connected gradings and the fundamental group 